\title{
DEVELOPMENT OF QUANTITATIVE STRUCTURE PROPERTY RELATIONSHIPS FOR PREDICTING THE MELTING POINT OF ENERGETIC MATERIALS
}

\author{
Jason A. Morrill \\ (corresponding author) \\ morrillj@william.jewell.edu \\ Department of Chemistry \\ William Jewell College \\ 500 College Hill \\ Liberty, MO 64068 \\ Edward F.C. Byrd \\ United States Army Research Laboratories \\ AMSRD-ARL-WM-BD \\ Aberdeen Proving Ground, MD 21005-5069
}




\section{Abstract}

The accurate prediction of the melting temperature of organic compounds is a significant problem that has eluded researchers for many years. The most common approach used to develop predictive models entails the derivation of Quantitative Structure-Property Relationships (QSPRs), which are multivariate linear relationships between calculated quantities that are descriptors of molecular or electronic features and a property of interest. In this report the derivation of QSPRs to predict melting temperatures of energetic materials based on descriptors calculated using the AM1 semiempirical quantum mechanical method are described. In total, the melting points and experimental crystal structures of 148 energetic materials were analyzed. Principal components analysis was performed in order to assess the relative importance and roles of the descriptors in our QSPR models. Also described are the results of $\mathrm{k}$ means cluster analysis, performed in order to identify natural groupings within our study set of structures. The QSPR models resulting from these analyses gave training set $R^{2}$ values of $0.6085\left(\mathrm{RMSE}= \pm 15.7{ }^{\circ} \mathrm{C}\right)$ and $0.7468\left(\right.$ RMSE $\left.= \pm 13.2^{\circ} \mathrm{C}\right)$. The test sets for these clusters had $R^{2}$ values of 0.9428 (RMSE $\left.= \pm 7.0^{\circ} \mathrm{C}\right)$ and $0.8974\left(\mathrm{RMSE}= \pm 8.8^{\circ} \mathrm{C}\right)$, respectively. These models are among the best melting point QSPRs yet published for energetic materials.

\section{Introduction}

The range in temperatures over which a substance melts is a physical property of fundamental importance. This is especially true in the energetic material community, where an unacceptably low melting point of a notional material can render it completely unfeasible for usage, regardless of any superior performance capability. When a substance 
undergoes a change in state from a relatively ordered solid to a disordered liquid the average thermal kinetic energy of the substance is sufficiently high so as to overcome the attractive forces between the constituents of the substance. The amount of thermal energy required to overcome the attractive forces depends on a great many factors including the types of electrostatic attractive forces between the constituents making up the substance, symmetry of the individual constituents, and efficiency with which the constituents are organized in the solid, just to name a few. In addition, so-called bulk effects, such as crystal defects, can affect the melting temperature of a substance. In common use the melting point range of a substance is a measure of its purity and also an indicator of useful temperature range for a substance. Prior knowledge of the melting point range is therefore an important consideration in designing new materials. Toward designing new materials a great many computationally derived mathematical models have been developed for predicting such physical properties as boiling points, octanol-water partition coefficients ${ }^{1}$, glass transition temperatures ${ }^{2}$, and melting points. ${ }^{1,3,4,5,6,7,8,9}$ While there have been many attempts to develop models for predicting melting points, to date most models have resulted in only moderately accurate predictive capability and very few have been developed specifically for energetic materials. Additionally, several attempts have been made to generate melting point models that are applicable to large numbers of structurally diverse compounds, it is generally known in the QSPR community that a more accurate model is produced when restricting the model to compounds of a common structure type.

Mitchell, et al, used an Artificial Neural Network (ANN) approach to explore hypothesized reasons explaining why the QSPR approach sometimes fails to develop good fits to experimental data and/or highly predictive multiple correlation models for various 
properties, including melting points. ${ }^{10}$ Using the Molecular Operating Environment and MMF94x molecular mechanics-based energy minimization the authors computed 567 2D and 3D descriptors for each molecule under investigation. The best model for each property was determined by using four different multiple correlation equation derivation algorithms: partial least squares, Random Forest, $k$ Nearest Neighbors, and Support Vector Machine. These algorithms were used to fit a pre-selected set of descriptors, chosen by the "Ant Colony Optimization" algorithm. The most predictive models were obtained for the octanol-water partition coefficient with an $R^{2}$ of 0.87 and the least predictive model was for the melting temperature with an $R^{2}$ of 0.46 . The authors suggested that having relatively few compounds with melting points at the extreme ends of the range in experimental data may have lead to the poor predictive capability of the model. They also suggested that thermal decomposition of some compounds on melting likely contributed to error in the experimental data that couldn't be accounted for in the regression models.

Karthikeyan, et al, employed an ANN approach to derive predictive models for a diverse set of 4173 organic molecules. ${ }^{3}$ The descriptor set that they employed was also diverse, having such descriptor types as physical quantities (charges, van der Waals volume, etc.), connectivity and topology indices, pharmacophore features (hydrogen bond donors/acceptors, charged partial surface areas, etc.). The charges and dipole moments were computed using PM3 ${ }^{11}$ and AM12 semiempirical quantum mechanical methods, respectively. The authors reduced the dimensionality of their data by performing principle components analysis (PCA). They found that the types of descriptors representing the most variance in the data could generally be categorized as those describing size, polarity, and sign of the surface area partial charge. Further, the authors found that models 
employing 2D descriptors outperformed both models based solely on 3D and those based on combinations of 2D and 3D descriptors. The 2D (best models) had coefficients of multiple determination of $\mathrm{R}^{2}=0.661$ for the training set and an RMSE of $48.0^{\circ} \mathrm{C}$. The corresponding test set gave an RMSE of $49.3^{\circ} \mathrm{C}$.

Given the documented difficulty in generating highly accurate melting point QSPRs, Preiss, et al, posed the question of whether it was even possible to generate a chemically reasonable, universal, and simple melting point predictive model. Their melting point model was based on the $1: 1$ salts of 520 organic compounds. ${ }^{8}$ Giving much consideration of the theoretical understanding of the phase change process of melting the authors ultimately derived a nine descriptor model. The descriptors were various types including computed entropy and enthalpy of solvation terms. The enthalpy of solvation was computed from geometries treated with the BP86 $13,14,15,16$ density functional theory (DFT) optimized geometries using the TZVP basis set ${ }^{17}$ and employing the COSMO solvation model ${ }^{18}$. Their model also included torsional flexibility, electrostatic, and molecular volume type descriptors. Their model provided an $\mathrm{R}^{2}$ value of 0.537 and an average error of $33.5^{\circ} \mathrm{C}$ or $9.3 \%$.

Salahinejad, et al, attempted to generate a generally useful model for the prediction of the enthalpy of sublimation $\left(\Delta H_{\text {sub }}\right)$ of small organic molecules. ${ }^{19}$ The authors fit a simple multiple linear regression model consisting of four preselected descriptors based on hydrophilicity and charged partial surface area descriptors (CPSA) using an artificial neural network (ANN) approach. The molecular basis set consisted of a very large and structurally diverse set of 8241 small organic molecules, which was divided into training and test sets of $80 \%$ and $20 \%$ of the total number of molecules, respectively. Using this 
approach they derived various QSPR models for $\Delta H_{\text {sub }}$ having $\mathrm{R}^{2}$ of 0.954 for the test set and $\mathrm{R}^{2}=0.950$ for the test set. Subsequently, they treated the predicted $\Delta H_{\text {sub }}$ values as a descriptor and incorporated additional descriptors by an artificial neural network approach to derive a QSPR model for melting points. The authors found it necessary to add 97 descriptors in multiple ANN-derived models to derive predictive QSPRs having $\mathrm{R}^{2}$ values that varied from 0.77 to 0.79 for the training sets and from 0.75 to 0.79 for the test sets.

Lazzus developed a QSPR that utilized descriptors from the results of calculations employing the PM3 semiempirical quantum mechanical method. ${ }^{20}$ His models were derived, first using CODESSA ${ }^{21}$ (Comprehensive Descriptors for Structural and Statistical Analysis) to compute and pre-analyze the descriptors to eliminate descriptors which were significantly intercorrelated or not significantly correlated with the experimental data. Following the filtering of descriptors Lazzus derived his predictive models using a Back Propagation ANN (BP ANN) method. For an eleven descriptor model fit to a training set of 260 compounds he found an average absolute deviation of $5.2 \%$ and for a 73 molecule test set the deviation was $4.8 \%$.

It is well known that QSPR models based on congeneric sets of structures generally give better results. By focusing their work more narrowly on compounds of a similar type, a number of investigators attempted to generate melting point QSPR models that would have to account for less variance in the experimental data. Guan, et al, derived QSPR models on the basis of structures that were optimized using the AM1 semiempirical quantum mechanical method..$^{22}$ The compounds under study were the bromide and chloride salts of various imidazoliums. Three sets of compounds were used and within 
each set there were training and test sets. The training sets consisted of 24,16 , and 40 compounds and the test sets consisted of a number of compounds that was $25 \%$ of the number in the corresponding training set. The authors carried out a comparison of Multiple Linear Regression (MLR) models obtained by Genetic Algorithms to those obtained by BP ANN methods. In general they found that the BP ANN models gave better results than the MLR models. The Mean Absolute Errors (MAEs) for each of the respective MLR model test sets were $20.52 \mathrm{~K}, 13.59 \mathrm{~K}$, and $21.95 \mathrm{~K}$. The MAEs for the BP ANN derived model test sets were $8.77 \mathrm{~K}, 4.98 \mathrm{~K}$, and $9.31 \mathrm{~K}$, respectively.

Brauner and Shacham examined the melting point of structurally homologous series of compounds, which varied only in the number of carbon atoms present in the molecule. ${ }^{23}$ Their multiple linear regression model was based only on functions of constitutional (number of carbon atoms) type descriptors and were regressed on melting points for varying series of long-chain hydrocarbon containing compounds ( $n$-alkanes , $n$-alkanoic acids, $n$-alkenes, $n$-mercaptans, and $n$-alkyl acetates). The average absolute relative errors reported for the different sets were between $1 \%$ and $9 \%$.

Holder and Liu derived a melting point QSPR for organosilicon compounds. ${ }^{24}$ Through a forward stepping multiple linear regression approach they developed a more comprehensive organosilicon melting point model, which was later refined for silanes only. All structures in Holder's work were modeled using the SAM1 semiempirical quantum mechanical method. ${ }^{25}$ The descriptors were obtained directly from these calculations or were calculated from quantities obtained in these calculations using CODESSA. For the general organosilicon model they found a six descriptor model for 97 training compounds having $\mathrm{R}^{2}=0.789$ and an RMSE of $50.45^{\circ} \mathrm{C}$. For the general organosilicon model an 
external test set of 32 compounds was found to have $\mathrm{R}^{2}=0.710$ and an RMSE of $42.44{ }^{\circ} \mathrm{C}$. A better fit was found for a more focused set of 62 structurally similar silanes. For this set Holder and Liu derived a 4 descriptor model having $\mathrm{R}^{2}=0.889$ and an RMSE of $34.09^{\circ} \mathrm{C}$ for the training set and an external test set $(\mathrm{N}=19)$ was found to have $\mathrm{R}^{2}=0.858$ and an RMSE $=38.44^{\circ} \mathrm{C}$. Holder's model was also refined by an iterative robust regression analysis. The descriptors in the obtained models were generally constitutional, electrostatic, and thermodynamic in nature and were found to be chemically sensible on the basis of theoretical considerations of melting behavior.

As we have just shown, there is a wide variety in the structural types that have been represented in general melting point QSPR models; however there are very few melting point models specifically for energetic materials. Moreover, the melting point QSPR models that do exist for energetic materials exhibit relatively weak predictive capability. Keshavarz and Pouretedal ${ }^{26}$ investigated a series of carbocyclic nitroaromatic energetic materials and report using a group additivity type approach. In their approach the only descriptors used were the atomic composition (number of hydrogen and nitrogen atoms), the specific pattern of substitution (orthovs. para), and the specific functional group contribution of the molecules. The exact nature of the descriptor, specific functional group contribution, was not described. In deriving the model, the descriptors were fit to the experimental data in the training set via simple linear regression. The training set consisted of 60 compounds, but the authors did not indicate what criteria were used in selecting the training set of compounds. The authors reported obtaining a mean percent error of $5.0 \%$. Although not reported, an analysis of their reported results revealed a 
mean absolute error of $18.1^{\circ} \mathrm{C}$, RMSE of $22.3^{\circ} \mathrm{C}$, and an $R^{2}$ of 0.81 . The range of error in the predicted values was from $-62.1^{\circ} \mathrm{C}$ to $53.8^{\circ} \mathrm{C}$.

Chakka, et al, reported on the development of a suite of programs that are directed at predicting the environmental fate and dispersion of various chemical compounds used in munitions via predictions of a set of physicochemical properties, including boiling and melting points, vapor pressure, aqueous solubility, octanol-water partition coefficient, carbon absorption, and Henry's Law constants. ${ }^{27}$ The authors used the Joback group contribution method combined with a correlation between boiling points and melting points $\left(\mathrm{T}_{\mathrm{m}}\right)$ to predict $\mathrm{T}_{\mathrm{m}}$ values. For the model there was no performance data reported, but the authors report very large deviations of predicted $\mathrm{T}_{\mathrm{m}}$ values from experiment for selected munitions-type compounds. For instance, for the four compounds tested the errors in predicted $\mathrm{T}_{\mathrm{m}}$ values ranged from $1.02{ }^{\circ} \mathrm{C}$ to $146.15^{\circ} \mathrm{C}$.

\section{Objective and General Approach}

The aim of this report is to use best practices as described in the preceding review of literature to develop a multilinear regression model that is capable of predicting the melting temperature of energetic materials with accuracy that is improved over those that have been reported previously. Previous models do not sufficiently demonstrate either high levels of accuracy or a sufficient generality in compound type. Although ANN models have been shown to generate melting point predictions that exhibit fits to experiment that are generally superior to linear models, ANN employ a very large number of descriptors and represent (by their very nature) a "black box" approach that does not readily lend itself to interpretation for design purposes.24,28 We desire the development of predictive 
models for the purpose of molecular design and have chosen to adopt a similar methodology to that of Holder and Liu. ${ }^{24}$

The work of Keshavarz and Pouretedal ${ }^{26}$ suggests that a correlation equation which exhibits a good fit between molecular descriptors and experimental melting points for energetic materials ought to be obtainable. The lack of statistical validation and external validation leaves one to wonder if their multiple correlation equation is over-fit to their training set data. Moreover, the lack of structural diversity in their training set further suggests that their model is not applicable to energetic materials that are not carbocyclic nitroaromatics. The approach described in this report is much less limited in the scope of energetic materials to which it applies and presents a balance between a good fit of descriptors to experimental data and predictive capability. While some have chosen to derive models for melting point prediction based solely on 2D molecular descriptors our approach is based on both 2D and 3D descriptors in an attempt to avoid introducing bias. Others have computed 3D descriptors for energy minimized molecular structures. However, our approach makes use of molecular geometries corresponding to the experimentally obtained crystal structures for the energetic materials in our study. We believe that using experimental crystal structures without energy minimization provides direct information about the actual 3D arrangement of atoms within the solid state of the material undergoing phase transition. We also believe that for computing descriptors this is the best representation of the material. Up to 683 descriptors were computed for each of the energetic materials under study. The descriptors belong to a diverse set of classes including topological connectivity, constitutional (atom count), geometrical (shape/volume), electrostatic (charged partial surface area), quantum mechanical (bond 
orders, orbital energy, etc.), and thermodynamic types of descriptors. The set of energetic materials studied also represents a diverse set of functionalities. Among the 148 energetic materials studied there were molecular and ionic solids, cyclic and acyclic molecules, and most classes of organic functional groups. This work represents a larger and more structurally diverse QSPR analysis for energetic material melting points than any published to date.

\section{Technical discussion}

\section{Molecular modeling}

All of the energetic materials under investigation were modeled using the AM1 semiempirical quantum mechanical method as implemented in the program, Ampac with Graphical User Interface. ${ }^{29}$ While it is customary to characterize an optimized geometry by means of a normal vibrational mode analysis, our approach was based on the experimental crystal structures and corresponding melting points obtained by the research group of Thomas Klapotke (the structures used and their melting points are listed in Table S1 in Supporting Information). ${ }^{30}$ We used these energy non-minimized structures as to not bias the descriptors with any errors that might arise from employing the semi-empirically derived structure. That said, our interest is in deriving an accurate QSPR model for the melting points of energetic materials. For such a predictive tool to be useful it must work for materials for which the arrangement of atoms in the solid state is unknown. Thus, validation was performed by testing on sets of compounds that were both in their experimental crystal structure geometry and as fully optimized and characterized stationary point geometries. The experimental crystal structures were modeled as the 
asymmetric unit cells bearing an overall neutral electronic charge and no periodic

boundary conditions were applied. Although some materials were found experimentally to exist as dimers in the asymmetric unit cell, we felt that all materials should be modeled as individual molecules in order to avoid introducing an assumption about the nature of information redundancy contained within the experimental structures.

\section{Descriptor calculation and QSPR derivation}

Descriptor and QSPR generation and analysis was performed with the program, CODESSA. CODESSA accepts the output from structural computations from Ampac and then both reads descriptors directly and computes descriptors from the data in the output file. Descriptors were not considered for generating multiple correlation equations if they did not have values, or if their values did not vary. Multiple correlation equations were generated using the Best Multilinear Regression (BMLR) algorithm in CODESSA. The function of the BMLR algorithm has been described previously. ${ }^{31}$ Briefly described, the algorithm is a forward stepping algorithm that begins by selecting an initial set of descriptors provided that they meet user definable criteria for sufficient correlation and sufficiently low intercorrelation with other descriptors. From this initial set of descriptors pair wise combinations are formed beginning with the descriptor with the highest $F$ value and the proceeding with the addition of the descriptor with the next highest $F$ value and so on. The set of equations having $F$ values higher that one-third of the highest $F$ value obtained are retained in each subsequent cycle of adding new descriptors, provided that the new descriptor is not significantly intercorrelated with any of the previously added 
descriptors. The addition of descriptors proceeds until a user definable maximum number of descriptors and equations is obtained.

\section{Results}

A ten-descriptor cutoff was chosen for a preliminary feasibility investigation on a training set consisting of 148 energetic materials. The best ten-descriptor model from CODESSA's BMLR simple least squares (LS) regression algorithm resulted in having a coefficient of multiple determination of $R^{2}=0.629$ and an $F$ value of $23.2(\mathrm{p}<0.001)$. In an attempt to improve the fit we conducted an iterative series of robust regression analyses in order to identify outliers in the experimental data. It has been shown that outlier identification can be successfully performed using least median squares (LMS) regression. ${ }^{32,33}$ We performed LMS regression analysis with the program, Stata $10 .{ }^{34}$ Following the LMS regression analysis the residuals were standardized by dividing the raw residual by a scale estimate, $s=\sqrt{s^{2}}$, where

$$
s^{2}=\frac{1}{n-p} \sum_{i=1}^{n} r_{i}^{2}
$$

for $n$ molecules, $p=2$ degrees of freedom, and where $r_{i}$ is the residual for the $\mathrm{i}^{\text {th }}$ experimental value. The standardized residuals, $r_{s}$, having values such that $\left|r_{s}\right|>2.5$ were the indentified as outliers and were removed from further analysis. Following this treatment a new training set was identified, the BMLR algorithm was used to derive a new multiple correlation equation, and the outlier removal process was repeated. In all, four cycles of outlier removal were performed, resulting in the removal of seven compounds and yielding a ten-descriptor model having $R^{2}=0.697$ and $F=29.9$. The multiple 
correlation equation and associated values are shown in Table 1. As indicated in Table 1 the preliminary multiple correlation equation represents a significant $(p<0.001)$ and good fit to the experimental data. The equation in Table 1 also exhibits some intercorrelation among the descriptors, as evidenced by four of the Variance Inflation Factors (VIFs) having values greater than 5.0. This over-fitting of descriptors is likely due to their inability to completely account for the experimental variance. Although the BMLR algorithm has a user-definable correction that accounts for intercorrelation amongst descriptors $\left(\mathrm{R}^{2}<0.6\right)$, the algorithm only compares descriptors in a pair-wise fashion. Instead of eliminating descriptors from further consideration on the basis of high intercorrelation we decided to perform cluster analysis on the descriptors in Table 1 in order to determine if the BMLR algorithm was using the descriptors to differentiate groups of compounds which have differing melting points for different reasons. In order to search for these clustered groups of compounds, k means cluster analysis was performed. ${ }^{34}$ In this analysis a random center point was chosen with a user-specified seed value for reproducibility. We arbitrarily specified that three groups were to be obtained and the measure of similarity or dissimilarity was the absolute distance from the center points. The analysis resulted in three energetic material clusters having 15 (cluster 1), 63 (cluster 2), and 62 (cluster 3) members each. The first group, cluster 1, was not retained for subsequent analysis due to its small size. From the two larger groups, test sets for validation were obtained by dividing the groups into property ranges. To obtain the ranges we performed a histogram analysis. According to Sturges' rule the number of classes of data in a group is given as $k=1+\log _{2} n$, where $n$ is the number of compounds in the 
group. For clusters 2 and 3 this resulted in 7.36 and 6.98 classes, respectively and these values were each

Table 1. Ten Descriptor Correlation Equation Obtained Using the Heuristic Algorithm.

\begin{tabular}{clccc}
\hline $\begin{array}{c}\text { Model } \\
\text { Parameters }\end{array}$ & \multicolumn{1}{c}{ Descriptor } & Coefficient & Student's $^{\mathrm{d}}$ & VIF $^{\mathrm{e}}$ \\
& & & $\mathbf{t}$ & \\
\hline & Avg. Coulson charge for an N atom & -153.3 & -6.75 & 4.15 \\
& Weighted positively charged surface area & -0.3399 & -4.46 & 5.89 \\
$R^{2}=0.697$ & Sum valence of all C atoms & 1.902 & 4.89 & 5.45 \\
$F=29.9^{\mathrm{a}}$ & Fractional negatively charged surface area & -588.0 & -9.74 & 2.98 \\
$R_{C V}^{2}=0.645^{\mathrm{b}}$ & Net Zefirov charge of all N atoms & -65.13 & -4.89 & 3.83 \\
$\mathrm{RMSE}^{\mathrm{c}}= \pm 23.2$ & LUMO + 1 energy & 23.54 & 7.71 & 2.20 \\
${ }^{\circ} \mathrm{C}$ & Avg. valence & 210.2 & 8.12 & 6.91 \\
$\mathrm{~N}=141$ & Avg. bond order & -417.3 & -5.58 & 6.40 \\
& Min. bond order & 78.74 & 6.35 & 2.94 \\
& Avg. information content & 36.64 & 3.55 & 1.27 \\
& Intercept & -99.64 & & \\
\hline
\end{tabular}

a) Fisher statistic value $(p<0.01)$. b) Leave-one-out cross-validated coefficient of multiple determination. $c)$ Root mean squared error. d) $p<0.001$ e) Variance inflation factor. 
rounded to 7 classes, or subgroups. In order to perform validation of the QSPR models for cluster 2 and cluster 3 the experimental melting point data for each of the clusters was then divided into 7 smaller ranges and the energetic material having the melting point closest to the midpoint of each range was removed and placed into the test set and the remainder was retained as the training set for validation. In situations where more than one melting point was equally close to the midpoint the choice for the test set was arbitrary. New QSPR models were then derived for the training sets for cluster 2 and cluster 3 energetic materials and used to predict values for the respective test sets of compounds. The resulting multiple correlation equation and associated values for clusters 2 and 3 are shown in Table 2. The names, experimental melting points, and identification numbers for the training and test materials in clusters 2 and 3 appear in Table 3. It is apparent that the cluster analysis resulted in training sets for clusters 2 and 3 are separated on the basis of energetic materials having higher melting points (cluster 2) and lower melting points (cluster 3). The range in melting points for cluster 2 is from $119^{\circ} \mathrm{C}$ to $247^{\circ} \mathrm{C}$ and the range for cluster 3 is from $18{ }^{\circ} \mathrm{C}$ to $122^{\circ} \mathrm{C}$. The descriptors appearing in Table 2 suggest that for both clusters molecular shape and intermolecular forces are important. The predictions for the test sets for each cluster of materials modeled as the non-optimized experimental crystal structures, as well as those materials modeled as fully optimized geometries are plotted in Fig. 1, where it is shown the models derived for clusters 2 (Fig. 1a) and 3 (Fig. 1c) modeled as experimental crystal structures exhibit good predictive capability. With one exception in each cluster group, the energetic materials melting point predictions are accurately ranked, as well. The predictions for the cluster 2 test set (modeled as optimized geometries) are slightly less accurate, but perform nearly as well as the prediction for the 
experimental crystal structures. The predictions for the cluster 3 test set modeled as optimized geometries 
Table 2. Correlation equation parameters and associated significance values for the multiple correlation equations for clusters 2 and 3 from the $k$ mean cluster analysis, derived after removing the test sets of compounds.

\begin{tabular}{|c|c|c|c|}
\hline Group & Descriptor & Coefficient & $\mathbf{V I F}^{\mathrm{e}}$ \\
\hline & Net Zefirov Charge of all N Atoms of a C-N bond & 677.6 & 1.38 \\
\hline Cluster 2 & Net Charge & -65.55 & 1.07 \\
\hline$R^{2}=0.6085$ & Number of C-C Bonds & 7.498 & 1.19 \\
\hline$F=12.7^{\mathrm{a}}$ & FNSA-3 (ESP charge) $)^{35,36}$ & -264.8 & 1.09 \\
\hline$R_{C V}^{2}=0.4073^{\mathrm{b}}$ & Maximum Valence & -160.6 & 1.52 \\
\hline$R_{A d j .}^{2}=0.5610^{c}$ & Balaban Index ${ }^{37,38}$ & 11.10 & 1.77 \\
\hline $\operatorname{RMSE}^{\mathrm{d}}= \pm 15.7^{\circ} \mathrm{C}$ & Intercept & 677.6 & \\
\hline \multicolumn{4}{|l|}{$\mathrm{N}=56$} \\
\hline \multirow{3}{*}{ Cluster 3} & Avg. Electrophilic Reactivity Index for an $\mathrm{N}$ atom & -239.9 & 1.40 \\
\hline & XY Shadow & -0.9930 & 1.71 \\
\hline & Number of C-O bonds & -8.301 & 2.22 \\
\hline$R^{2}=0.7468$ & Sum Bond Order for all C Atoms & 2.314 & 3.23 \\
\hline$F=19.8^{\mathrm{a}}$ & Sum One-electron reactivity index for all $\mathrm{C}$ atoms & -226.0 & 1.06 \\
\hline$R_{C V}^{2}=0.6346^{\mathrm{b}}$ & Min. Valence & -249.4 & 1.43 \\
\hline$R_{A d j .}^{2}=0.7091^{c}$ & Avg. Electrophlic Reactivity Index for an $\mathrm{H}$ atom & 200.4 & 1.13 \\
\hline $\operatorname{RMSE}^{\mathrm{d}}= \pm 13.2^{\circ} \mathrm{C}$ & Intercept & 340.9 & \\
\hline $\mathrm{N}=55$ & & & \\
\hline
\end{tabular}

a) Fisher statistic value $(p<0.01)$. b) Leave-one-out cross-validated coefficient of multiple determination. $c)$ Adjusted coefficient of multiple determination. d) Root mean squared error. e) Variance inflation factor. 


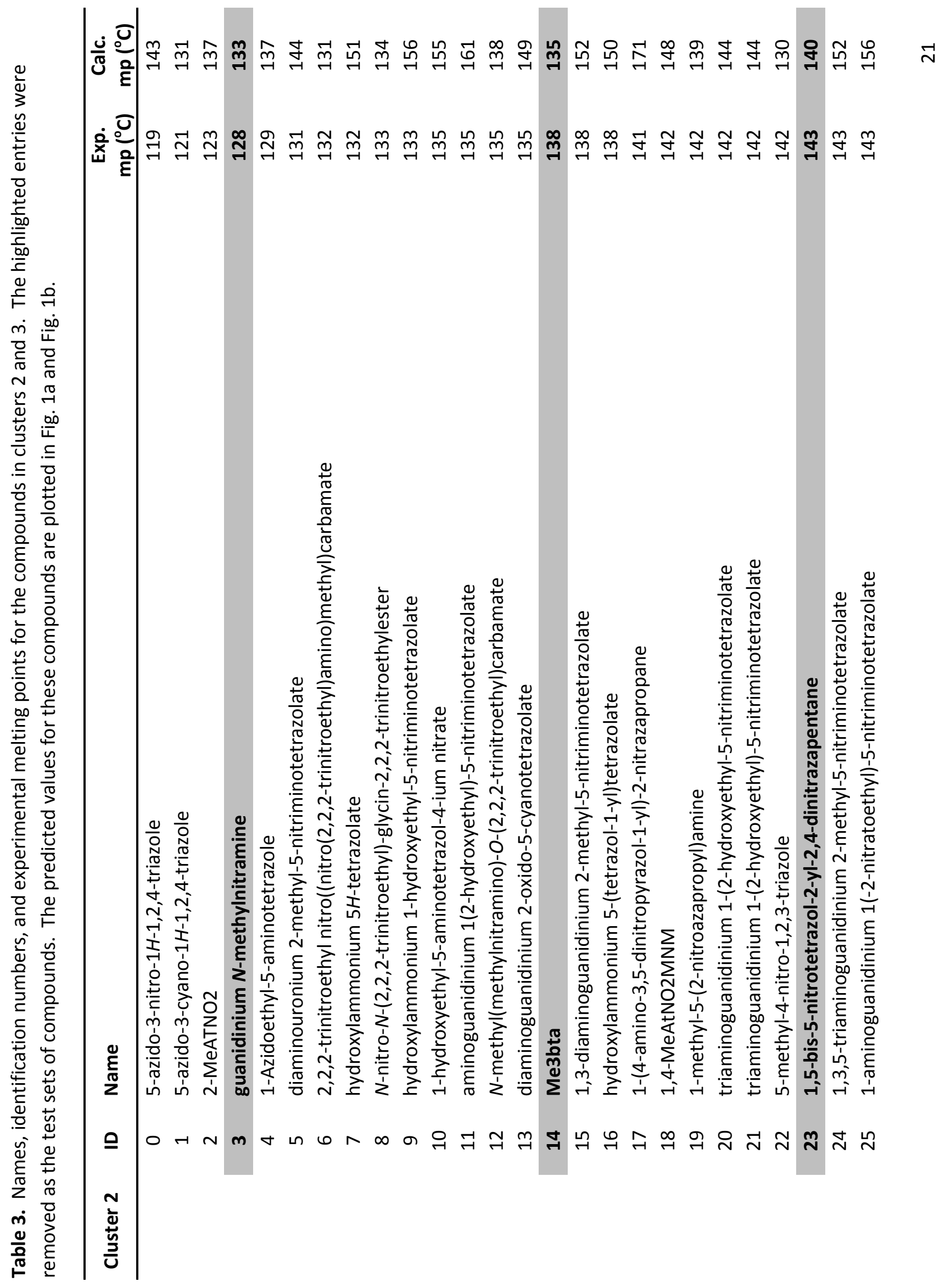




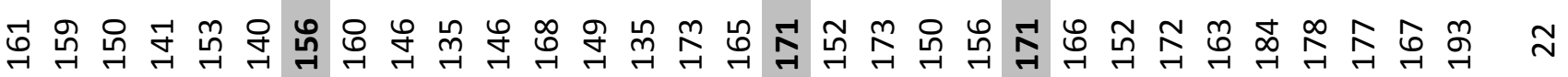

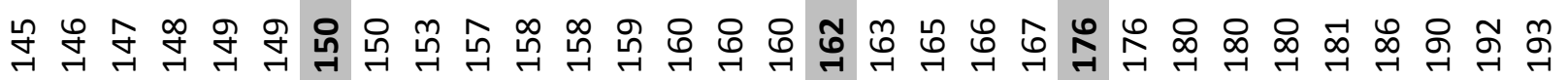

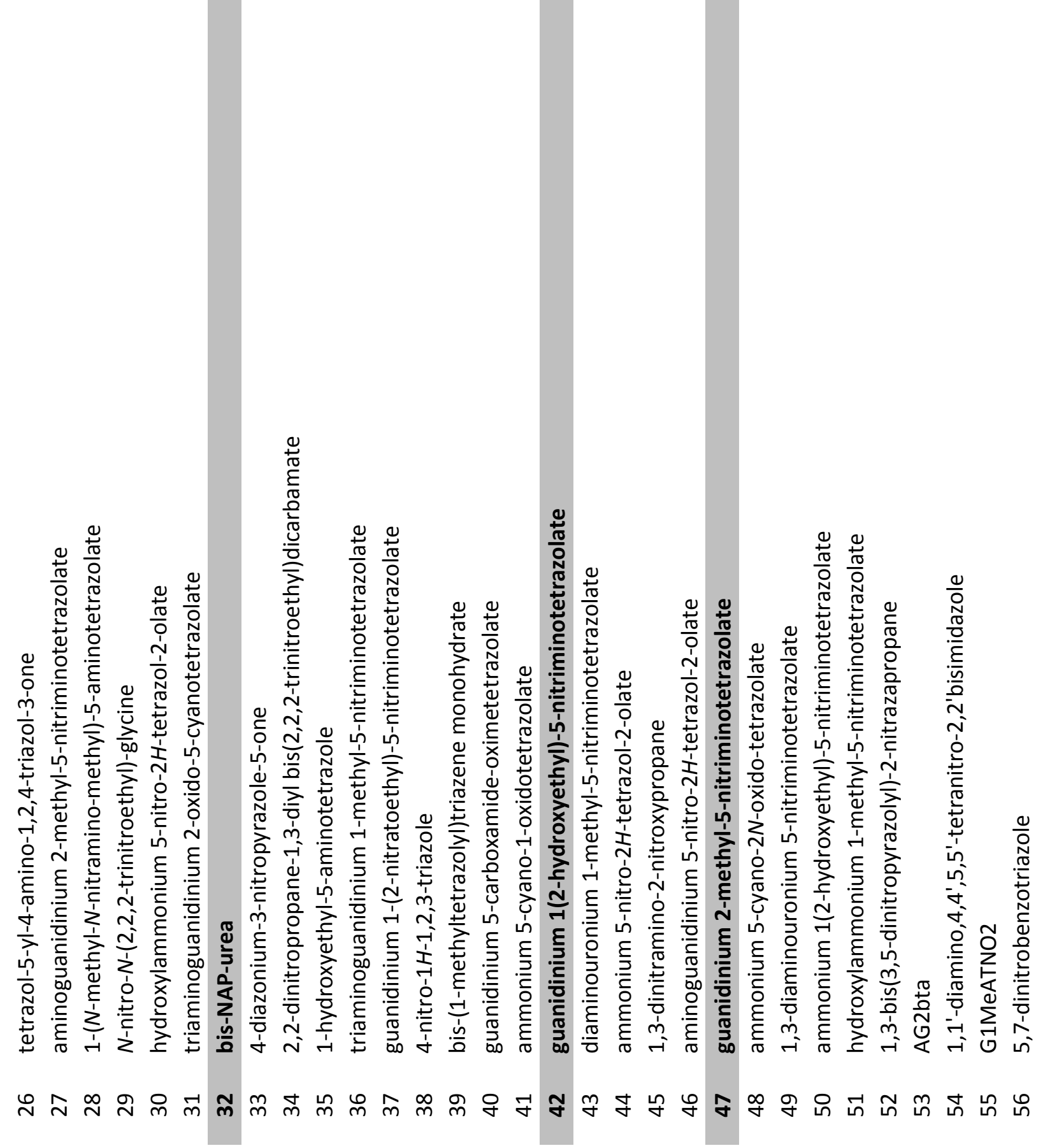




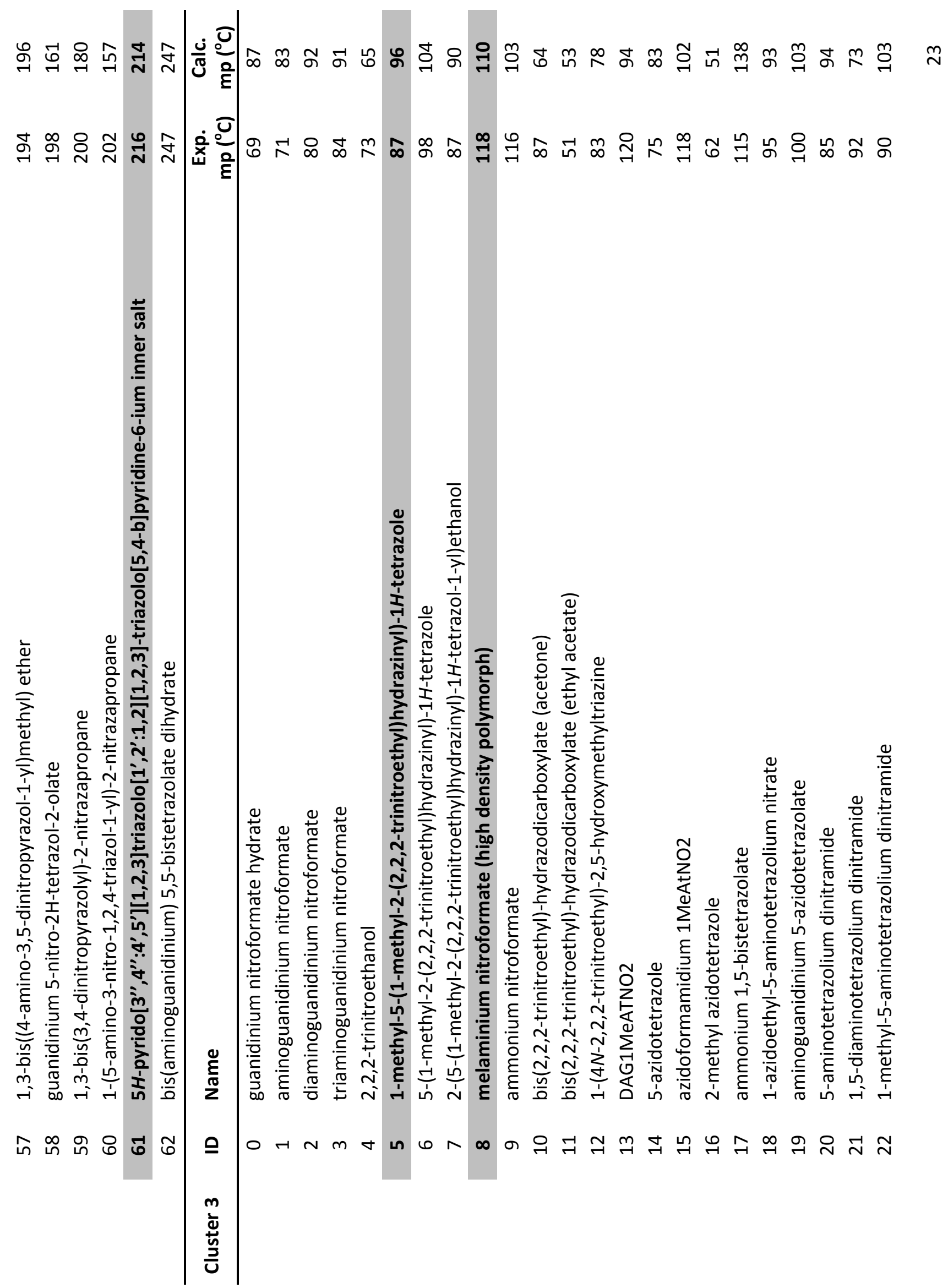




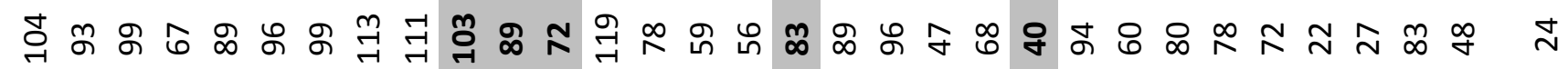

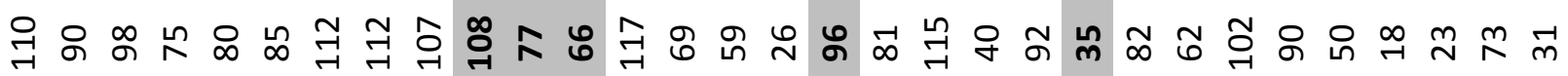

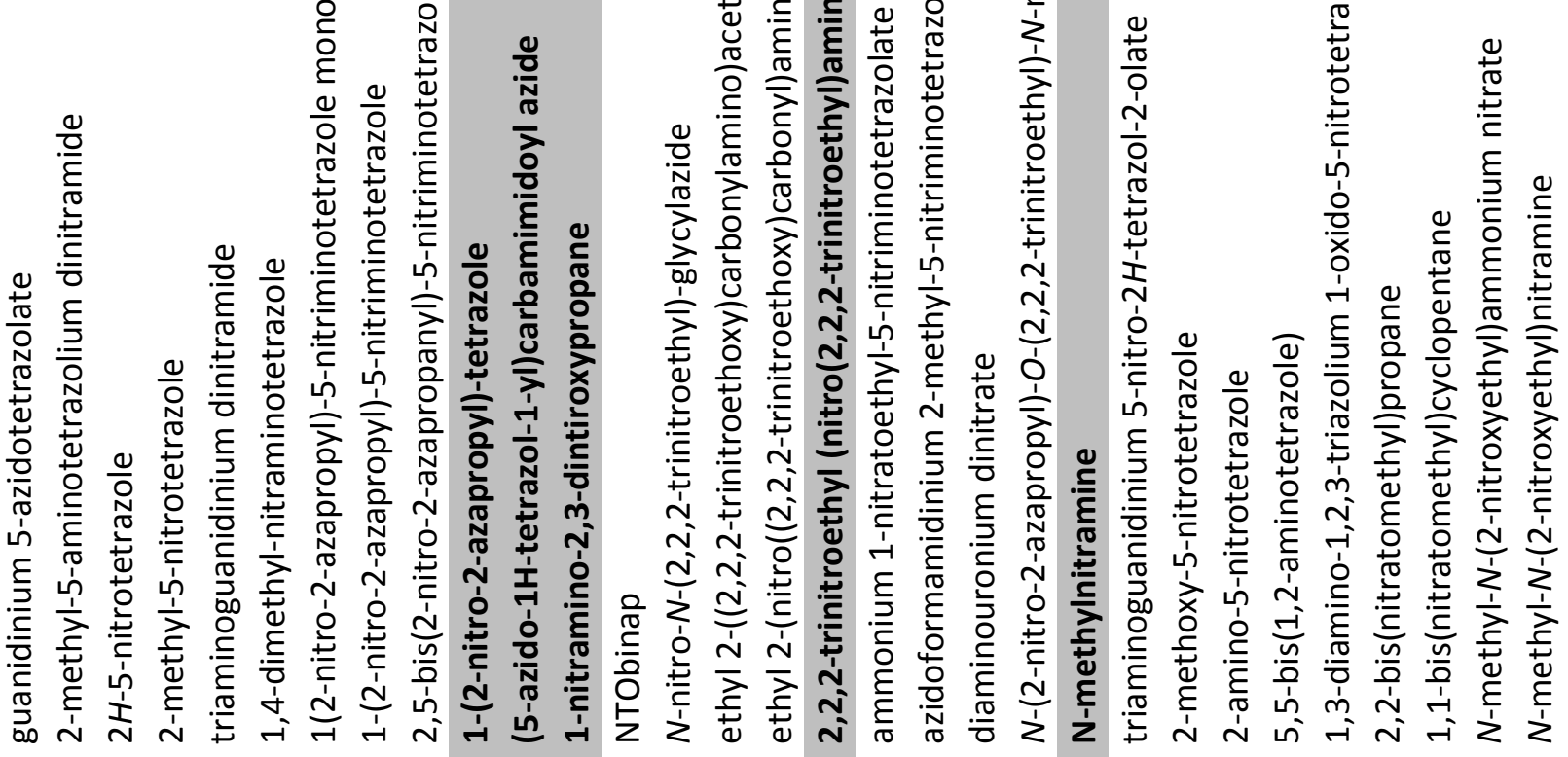

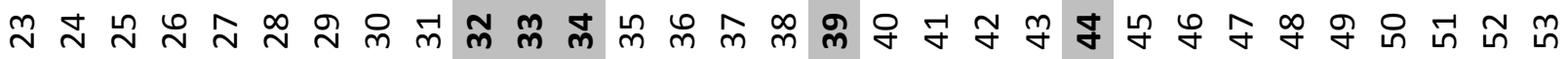




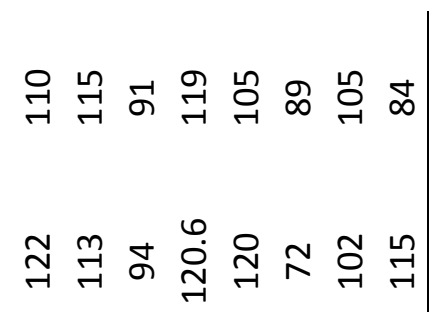

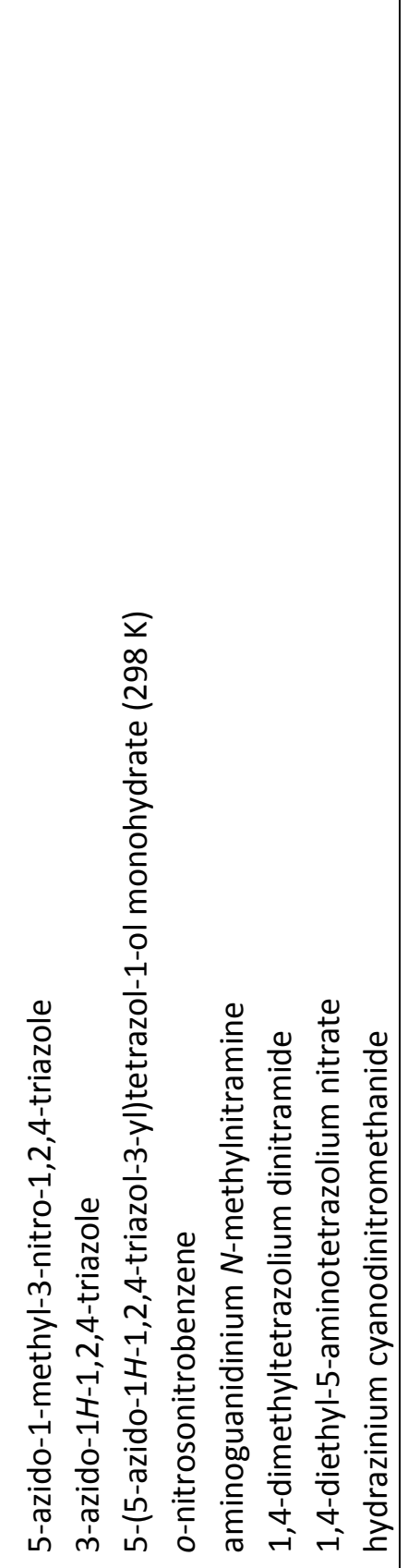

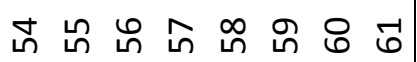




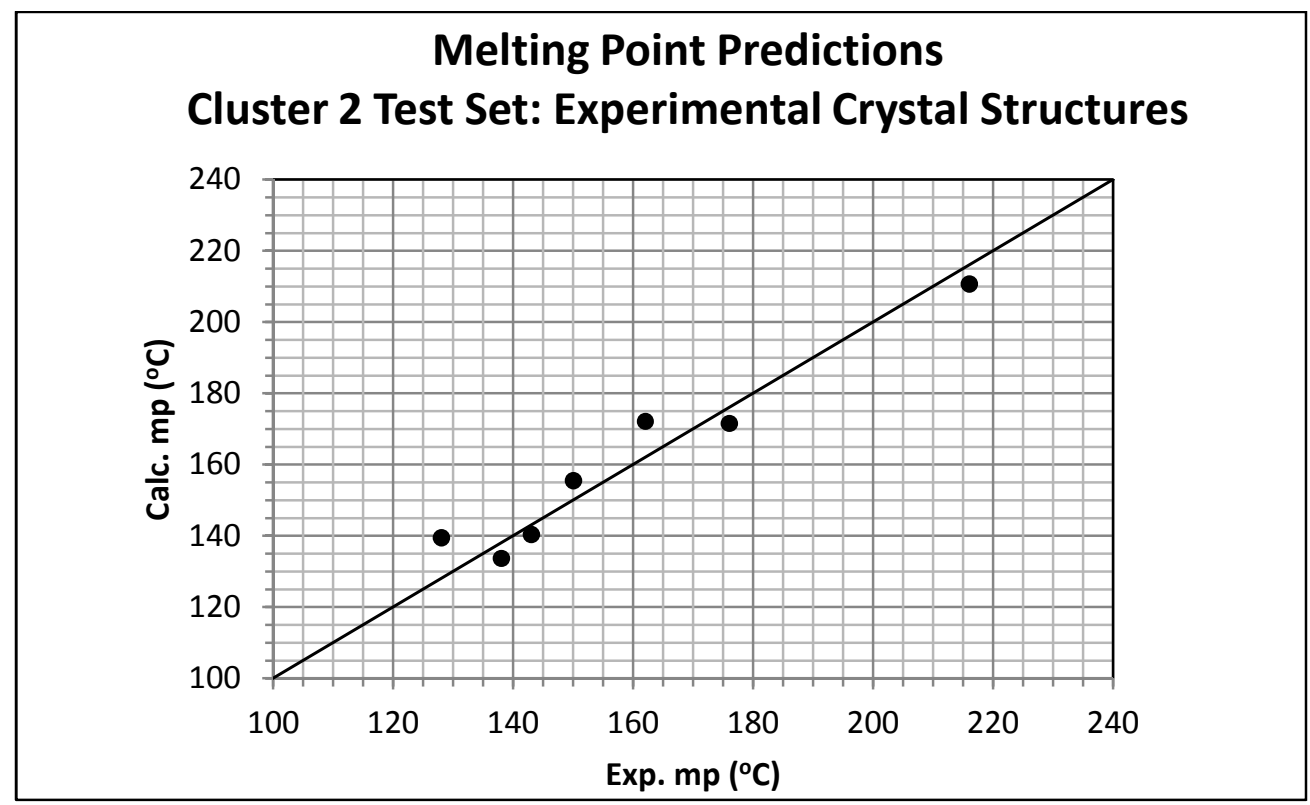

Figure 1a. Calculated vs. experimental melting points for the cluster 2 test set of materials, modeled as experimental crystal structures. For the prediction, $R^{2}=0.9428$ and $R M S E= \pm 7.0^{\circ} \mathrm{C}$.

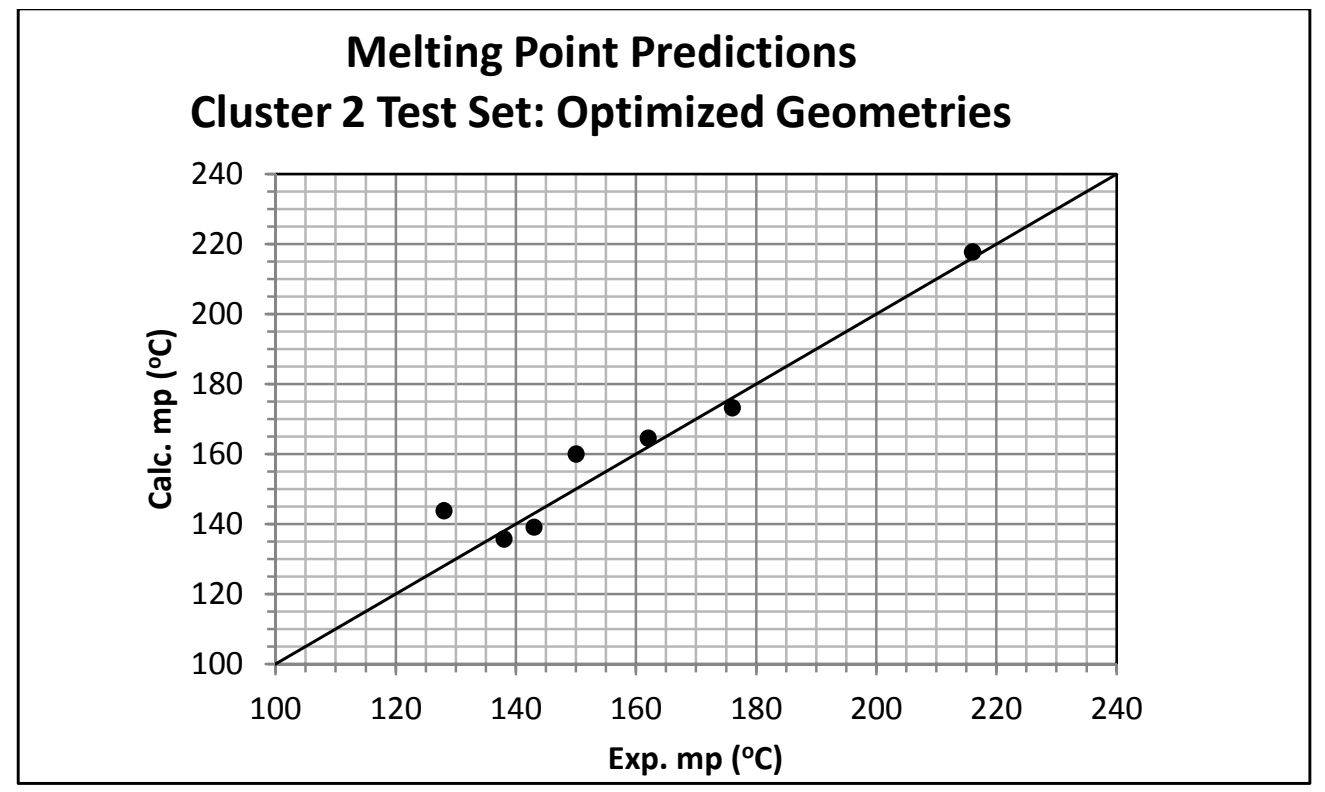

Figure 1b. Calculated vs. experimental melting points for the cluster 2 test set of materials, modeled as fully optimized geometries. For the prediction, $\mathrm{R}^{2}=0.9391$ and $\mathrm{RMSE}= \pm 7.5^{\circ} \mathrm{C}$. 


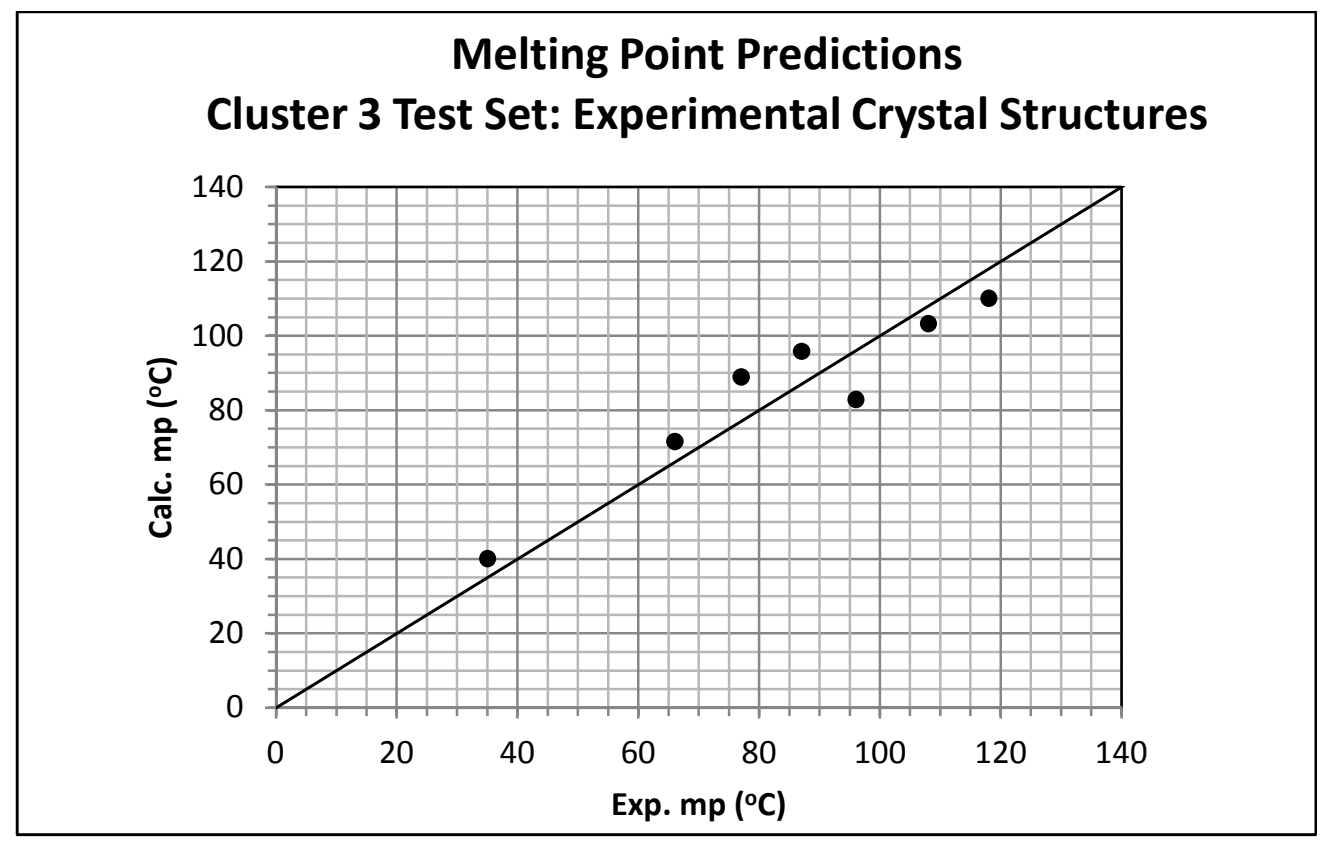

Figure 1c. Calculated vs. experimental melting points for the cluster 3 test set of materials, modeled as experimental crystal structures. For the prediction, $R^{2}=0.8974$ and $R M S E= \pm 8.8^{\circ} \mathrm{C}$.

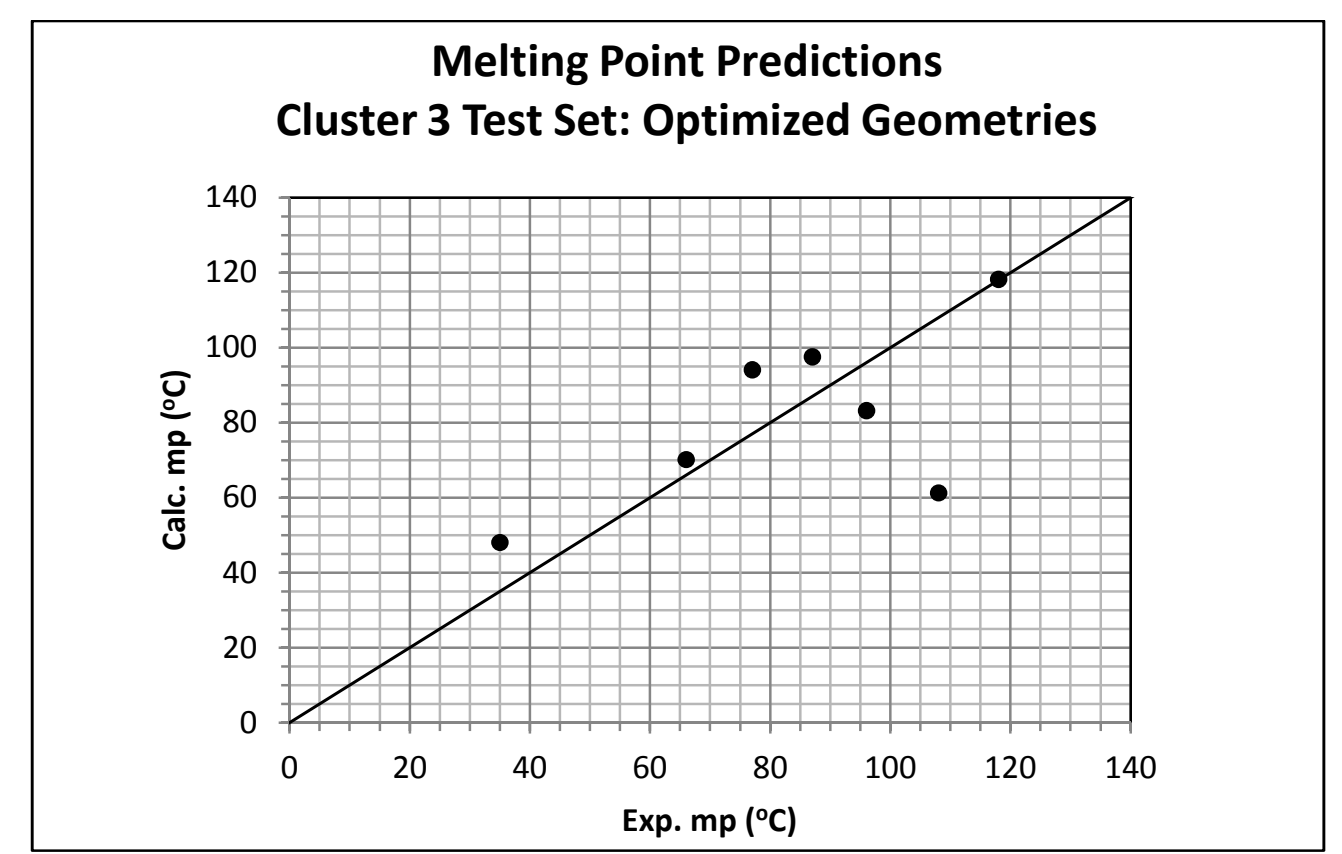

Figure 1d. Calculated vs. experimental melting points for the cluster 3 test set of materials, modeled as experimental crystal structures. For the prediction, $R^{2}=0.4193$ and $R M S E= \pm 20.5^{\circ} \mathrm{C}$. 
exhibits similar performance to the experimental structure predictions, with the exception of one material. The material, 1-(2-nitro-2-azapropyl)-tetrazole, has a predicted melting point for the fully optimized geometry that is $46.7^{\circ} \mathrm{C}$ lower than its experimental melting point. The prediction for the experimental crystal structure is only $4.6^{\circ} \mathrm{C}$ lower than the experimental melting point. Comparison of the experimental crystal structure with the optimized geometry for this material reveals that while the experimental crystal structure is nearly planar (deviating approximately $1.8^{\circ}$ out of plane) at the 2 -azapropyl nitrogen, the AM1 optimized geometry is pyramidal (deviating $37.5^{\circ}$ out of plane) at this position. This structure for this material was also optimized at the B3LYP/6-31G+(d,p) level, giving slight improvement in the geometry as the result deviated from planarity by $28.8^{\circ}$ and this geometry gave an improved predicted melting point of $83.9^{\circ} \mathrm{C}$, lower than the experimental melting point by $24.6^{\circ} \mathrm{C}$.

To better ascertain the roles of descriptors in each of the correlation equations we performed principal components analysis (PCA) on the QSPR models for the training sets for clusters 2 and 3 . Over $60 \%$ of the variance was explained in the first three principal components for both clusters 2 and 3. Satisfied with the amount of variance explained, our analysis was limited to the first two components. The results of the principal component analysis for cluster 2 are plotted in Fig. 2. From a comparison of Fig. 2 with corresponding melting points, it is apparent that the materials with higher melting points are oriented more to the periphery of the score plot. An analysis of the predicted scores from the PCA for cluster 2 shows that compounds having a predicted score (for component 1 ) of between -1.0 and 1.0 exhibit an average melting point of $154^{\circ} \mathrm{C}$, whereas those having a predicted score outside of this range have an average melting point of $161^{\circ} \mathrm{C}$. We interpret this 


\section{PCA Score Plot for Cluster 2}

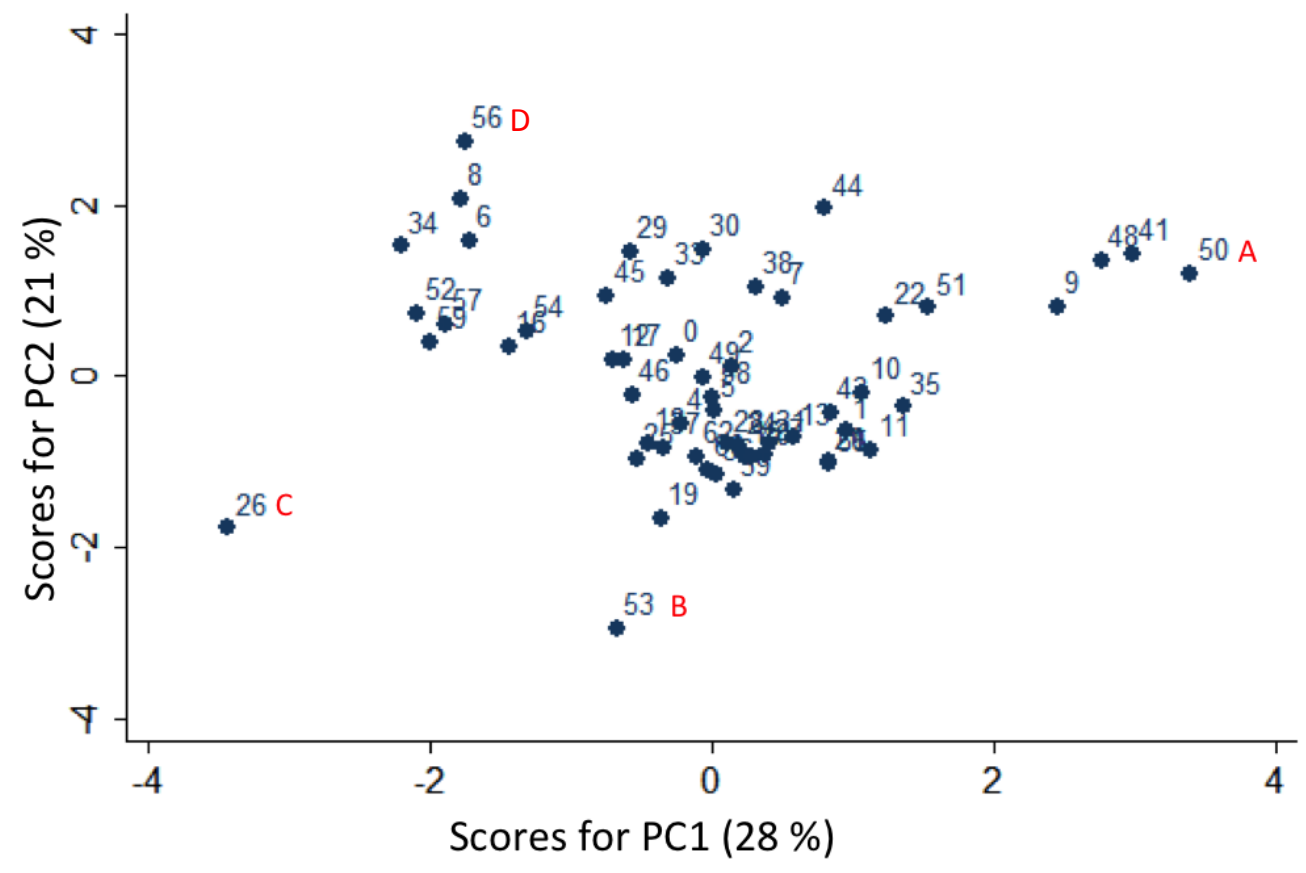

\section{PCA Loading Plot for Cluster 2}

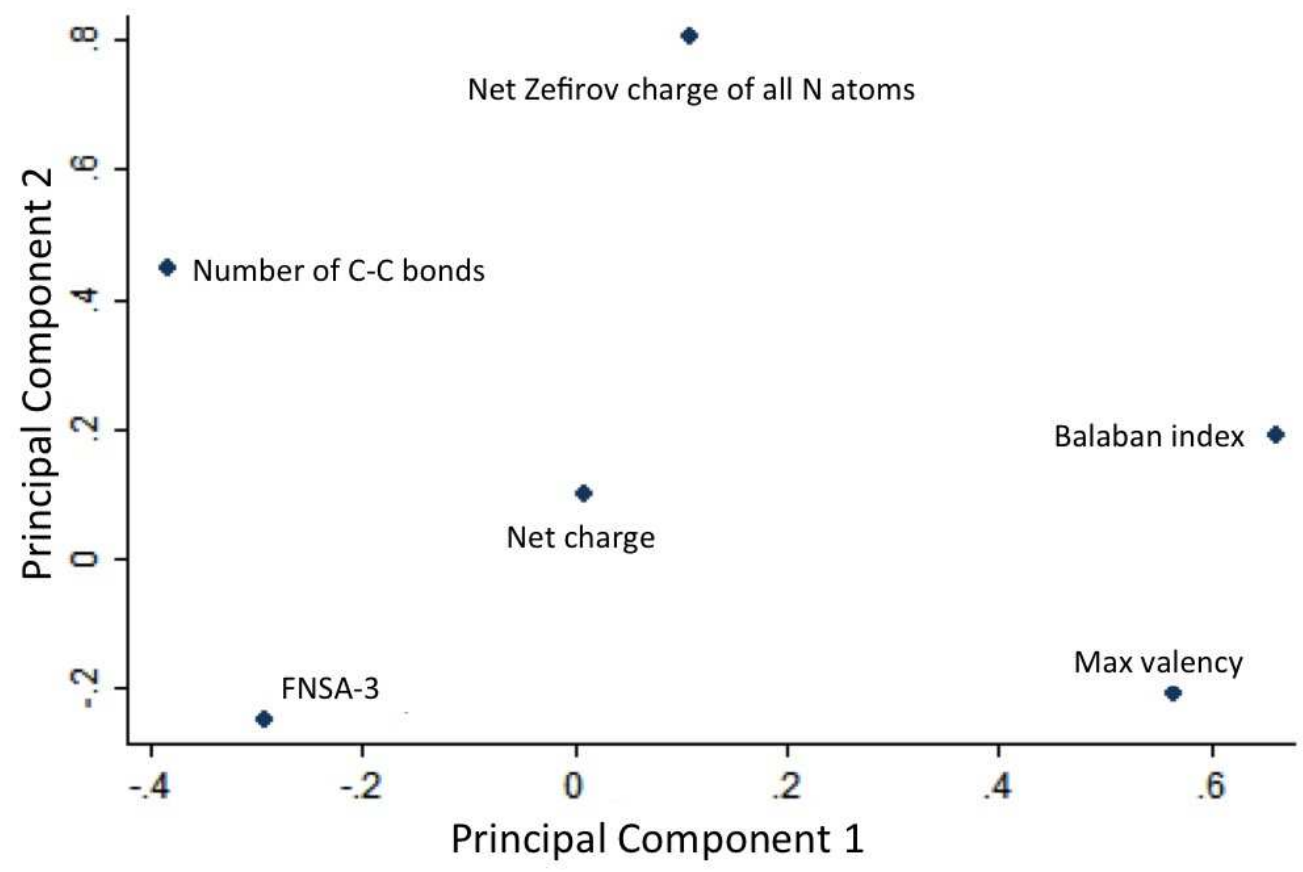

Figure 2. Score (top) and loading (bottom) plots from the principal components analysis of cluster 2. 
behavior as the model accounting for variance in the melting points in these materials arising for differing reasons due to the high degree of structural variation in the training set of energetic materials. The following examples illustrate this point. In the PCA score plot (Fig. 2) for cluster 2, ammonium 1(2-hydroxyethyl)-5-nitriminotetrazolate (compound A in Fig. 3) appears to the far right and is associated most closely with the Balaban index (J) descriptor (see loading plot in Fig. 2). The Balaban index is computed from molecular graph theory as $J=\left(\frac{q}{\mu+1}\right) \sum_{i, j}^{q}\left(s_{i} s_{j}\right)^{-\frac{1}{2}}$

where $q$ is the number of edges in the molecular graph, $\mu=q-n+1$ is the cyclometric number with n vertices in the graph, and $s_{i}$ and $s_{j}$ are the distance sums (from the distance matrix) obtained from summing the row $i$ (or $j$ ) and column $i$ (or $j$ ) of the distance matrix elements for the molecule. Essentially, the Balaban index is a measure of the compactness due to branching within a structure. A more compact molecule will have smaller values for the elements in the distance matrix because the number of bonds between the atoms within the molecule (the distance) is smaller. For a more linear molecule the Balaban index takes on larger value and in the cluster 2 QSPR model describes molecules that have higher melting points due to greater van der Waals forces. The energetic material, AG2bta (compound B in Fig. 3), is more associated with the net charge descriptor as indicated in the loading plot in Fig. 2. This compound has a relatively high nitrogen composition compared to other energetic materials in this investigation. The higher nitrogen content 


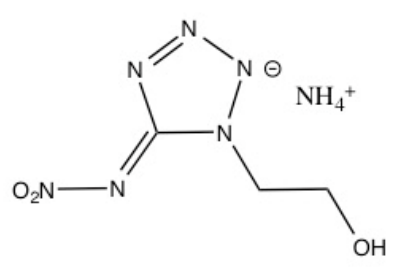

A

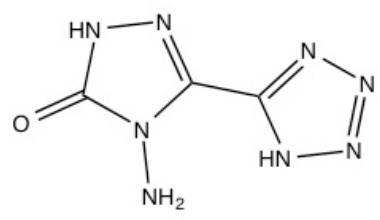

C

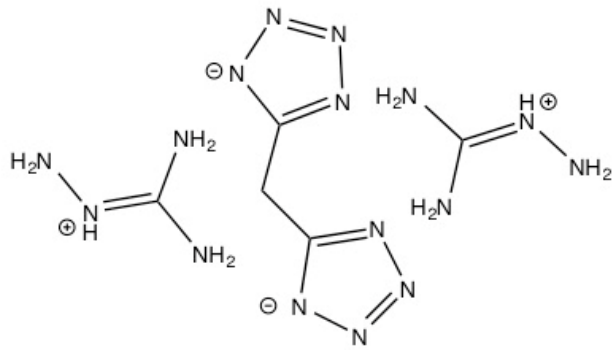

B

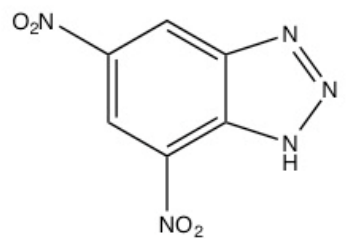

D

Figure 3. Structures discussed in the interpretations of the principal components analysis of cluster 2. 
leads to a greater charge being carried by the atoms in these types of materials and greater ionic character in their intermolecular interactions. The importance of the net charge descriptor suggests that for compounds like AG2bta intermolecular interactions are more ionic and stronger in nature. The stronger interactions in compounds like AG2bta lead to higher melting points in these materials. Energetic material, tetrazol-5-yl-4-amino-1,2,4triazol-3-one (compound C in Fig. 3), most closely associates with the FNSA-3 descriptor (see loading plot in Fig. 2). This descriptor has a negative component loading in the first principal component (PC1), meaning that the more negatively charged surface area in these materials likely leads to more and stronger hydrogen bonding interactions in these materials and consequently higher melting points. In compound C hydrogen bonding interactions are evident from the crystal structure of this material (see Fig. 4) and aid in this interpretation. Finally, the number of C-C bonds descriptor is also closely associated with compounds like 5,7-dinitrobenzotriazole (compound D, Fig. 3). The C-C bonds that are present in the higher melting materials tend to be $\pi$ bonds and the $\mathrm{C}-\mathrm{C}$ bonds in the lower melting materials tend to be $\sigma$ bonds. We interpret this descriptor as indicating that the greater presence of $\mathrm{C}-\mathrm{C} \pi$ bonds giving rise to greater planarity, more efficient packing, and greater van der Waals forces to be overcome as the material melts.

The results of PCA for cluster 3 are plotted in Fig. 5. Inspection of Fig. 5 suggests that the descriptors are again accounting for a wide degree of structural variation, but for a group of energetic materials having lower melting points. The energetic materials 2,2bis(nitratomethyl)propane and 1,1-bis(nitratomethyl)cyclopentane (compounds E and F, respectively [Fig. 6]) closely associate with the minimum valence descriptor. The atoms possessing the minimum valence in these materials are circled in Fig. 6. We interpret this 


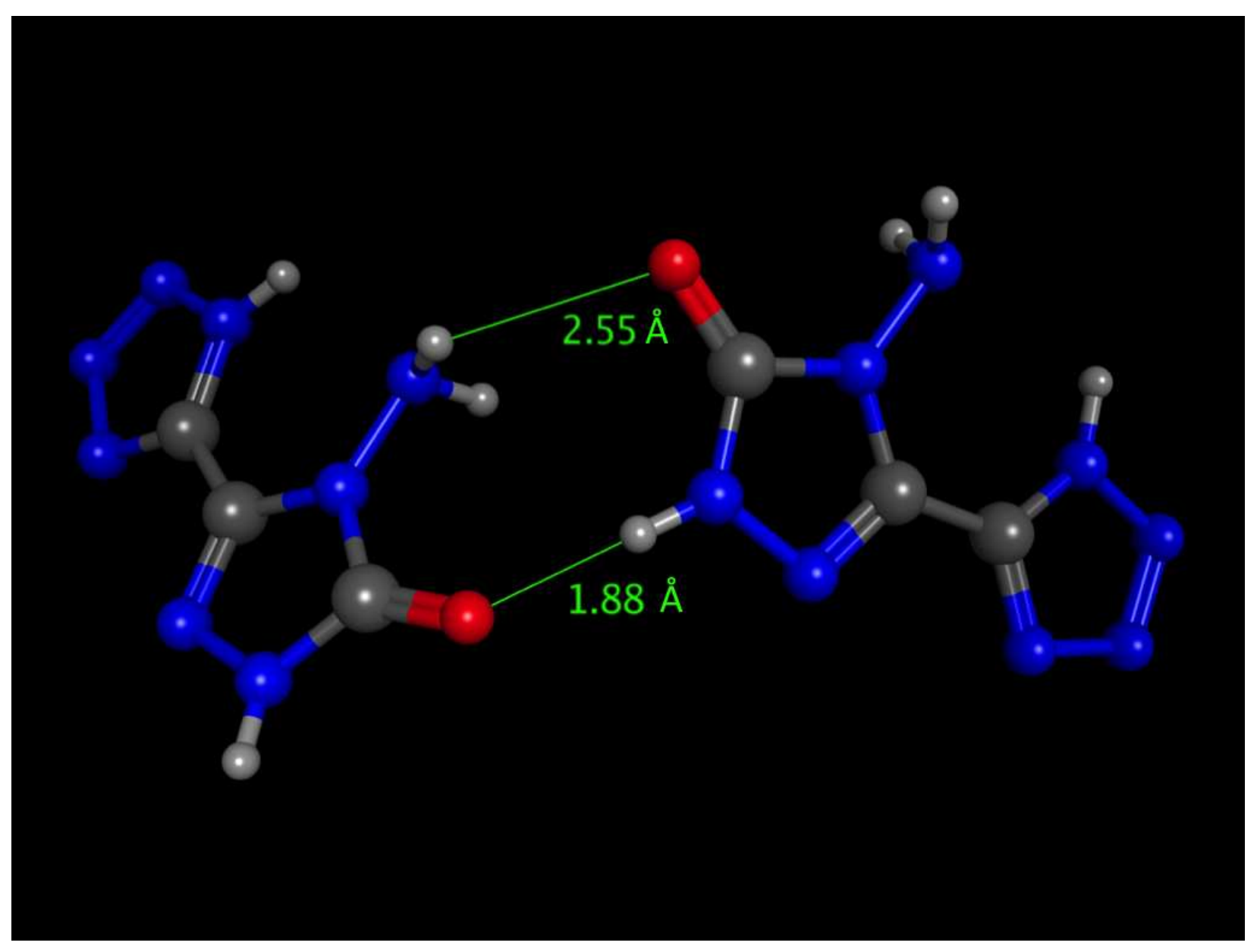

Figure 4. Crystal structure of tetrazol-5-yl-4-amino-1,2,4-triazol-3-one (compound C) with the hydrogen bonds indicated. 

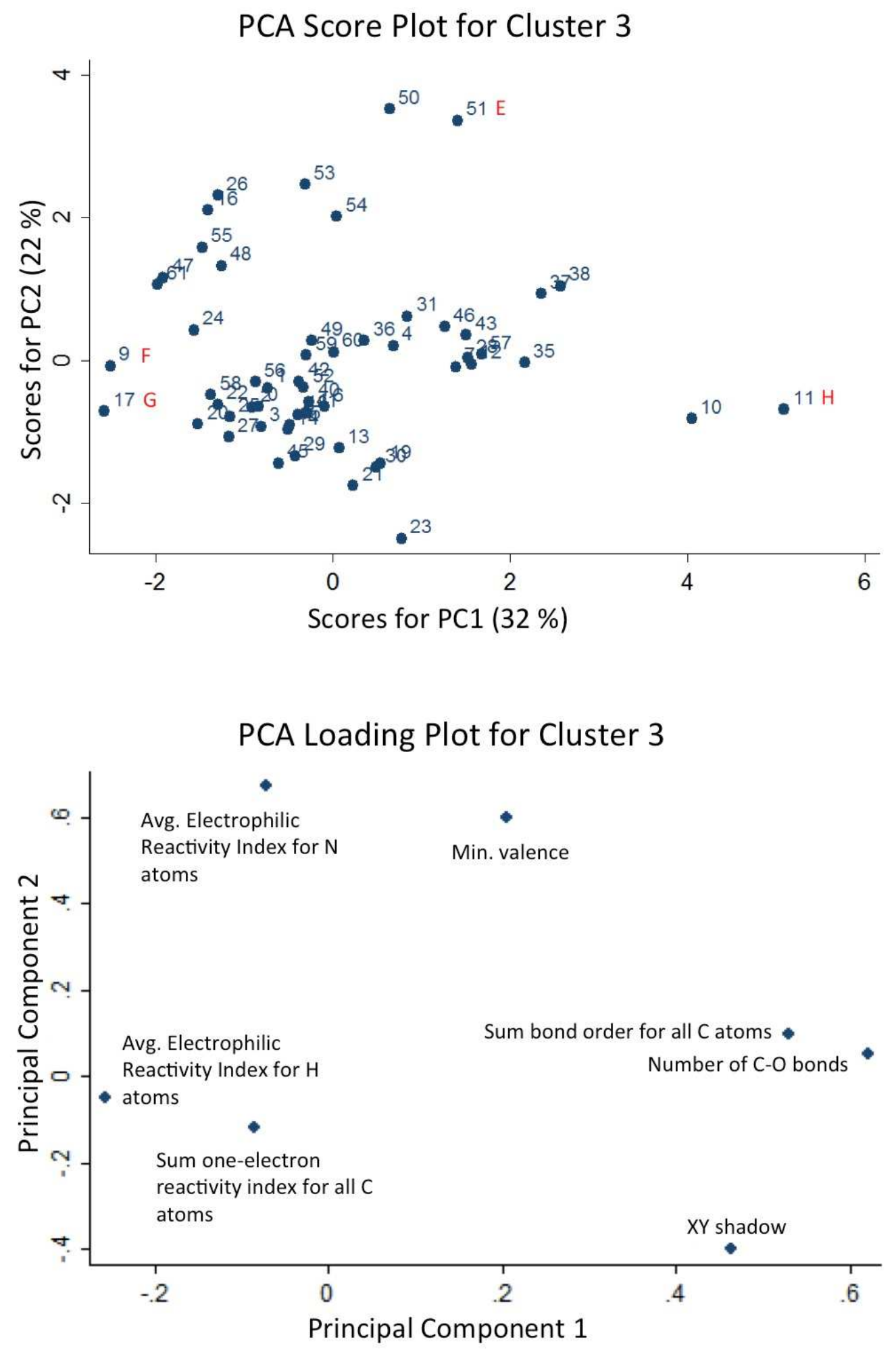

Figure 5. Score (top) and loading (bottom) plots from the principal components analysis of cluster 3. 


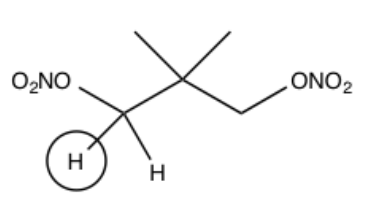

E

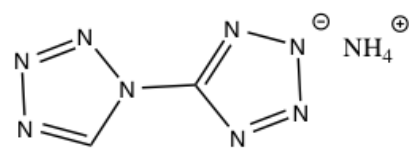

H

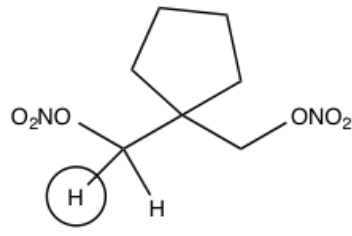

$\mathbf{F}$

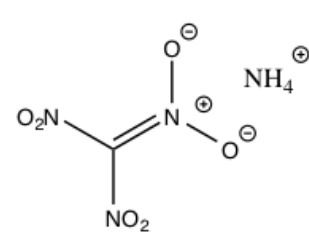

G<smiles>O=C(NNC(=O)OCC([N+](=O)[O-])([N+](=O)[O-])[N+](=O)[O-])OCC([N+](=O)[O-])([N+](=O)[O-])[N+](=O)[O-]</smiles><smiles>CCOC(C)=O</smiles>

I

Figure 6. Structures discussed in the interpretations of the principal components analysis of cluster 3. 
descriptor as accounting for weak $\mathrm{CHO}$ hydrogen bonding, or weak dipole-dipole interactions, between these materials and the lower melting points for these materials as arising from little thermal being required to overcome these weak attractive forces. The average electrophilic reactivity index for a nitrogen atom and a hydrogen atom are derived from Fukui molecular orbital theory ${ }^{39}$ and are calculated according to the following formula,

$$
E_{a}=\sum_{j \in A} \frac{c_{j L U M O}^{2}}{\left(\varepsilon_{\text {LUMO }}+10\right)}
$$

where the atomic orbital coefficients to the Lowest Unoccupied Molecular Orbital (LUMO) $c$, are centered on atom, $\mathrm{A}$ ( $\mathrm{N}$ or $\mathrm{H}$ in this case), and $\varepsilon$ is the LUMO energy. The Pearson product moment correlations for these descriptors for correlation with respect to the melting temperatures for the materials in the cluster 3 training set are -0.53 for an $\mathrm{N}$ atom and 0.26 for an $\mathrm{H}$ atom, thus it appears these descriptors are describing hydrogen bonding in two different ways. The energetic materials most closely associated with this descriptor are ammonium nitroformate and ammonium 1,5-bistetrazolate (compounds $\mathrm{G}$ and $\mathrm{H}$, respectively, see Fig. 5 and Fig. 6). The effect brought about by this interaction is likely due to the presence of the ammonium counter-ion helping to establish a hydrogen bonding network in these types of materials. This idea is supported by the relatively high melting points exhibited for these materials $\left(116^{\circ} \mathrm{C}\right.$ for compound $\mathrm{G}$ and $115^{\circ} \mathrm{C}$ for compound $\mathrm{H}$ ) and by the hydrogen bonds evident from the crystal structures for these compounds, shown in Fig. 7. In this model the two descriptors, sum of bond orders of all $\mathrm{C}$ atoms and XY shadow (a projection along the first and second axes of principal axes of inertia), are 

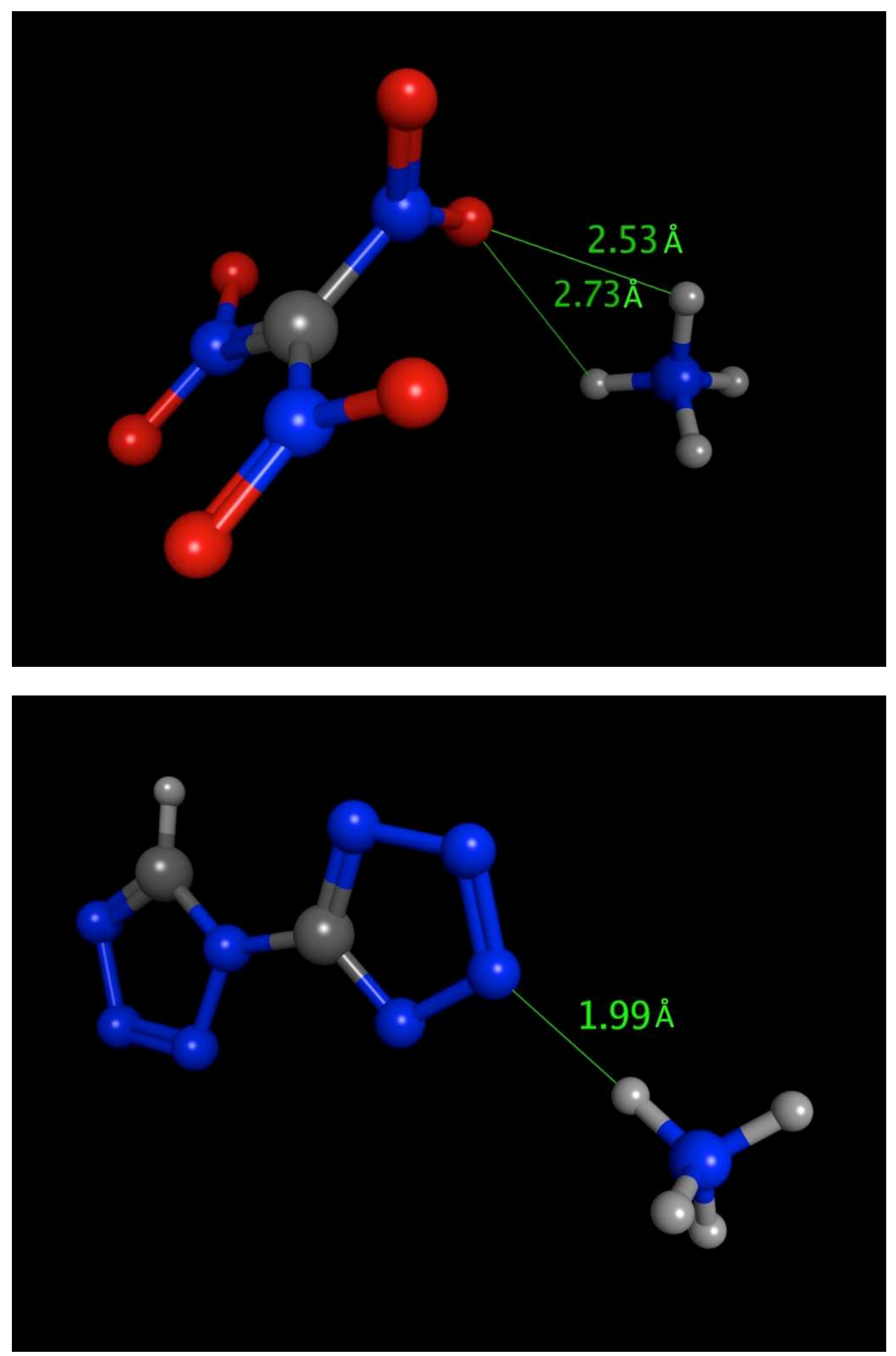

Figure 7. Crystal structures of ammonium nitroformate (top) and ammonium 1,5-bistetrazolate (bottom) (compounds $\mathrm{G}$ and $\mathrm{H}$, respectively) with the hydrogen bonds indicated. 
descriptors of molecular flexibility and shape, respectively. These descriptors have similar loadings in the principal components analysis (along PC1) and they describe similar melting behaviors. This idea is exemplified by the energetic material, bis $(2,2,2-$ trinitroethyl)-hydrazodicarboxylate (co-crystallized with ethyl acetate, compound I), which has an intermediate melting point in cluster 3 of $51^{\circ} \mathrm{C}$. The linear nature of this material is evident when viewing the material' $\mathrm{s}$ crystal structure oriented along the $\mathrm{X}$ and Y principal axes (see Fig. 8). The linear nature of this molecule likely promotes more van der Waals interactions and somewhat higher melting points than energetic materials of similar mass, but lower linearity. A higher melting point would also be supported by a lower degree of conformational mobility as described increased bond order in bonds between carbon atoms.

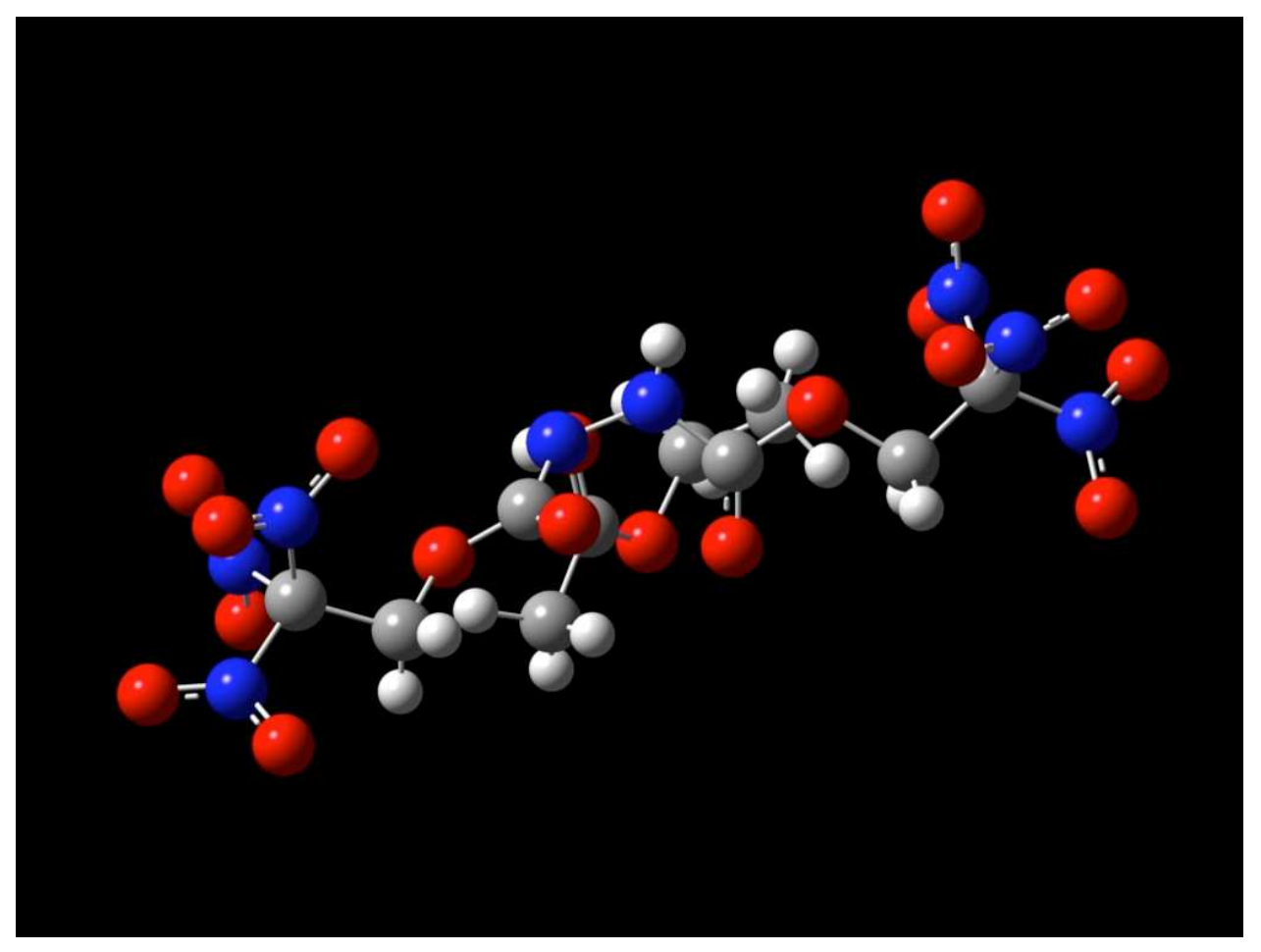

Figure 8. Crystal structure of bis(2,2,2-trinitroethyl)-hydrazodicarboxylate (co-crystallized with ethyl acetate, compound I oriented along the $\mathrm{X}$ and $\mathrm{Y}$ principal axes of inertia to illustrate the $\mathrm{XY}$ shadow descriptor. 


\section{Conclusions}

We have derived QSPR models for the melting points of a large number of structurally diverse energetic materials that are as predictive as, or more predictive than, QSPR models that have been previously published for such materials. Moreover, the descriptors resulting from QSPR derivation using CODESSA are chemically reasonable and reflective of what is known about the melting process. Although our models are predictive their accuracy could potentially be improved by further investigation into more structurally similar sets of energetic materials. As suggested by our tests of predictive capability, the level of theory which best reproduces experimental crystal structures will likely result in predictions that more closely represent experiment. Finally, incorporation of descriptors and perhaps algorithms beyond those derived with CODESSA may result in greater predictive capability.

\section{Acknowledgements}

This work was performed under the auspices of the U.S. Army Research Office Scientific Services Program administered by Battelle Memorial Institute, Contract No. W911NF-11-D0001. The authors would like to thank Thomas Klapötke and Jorg Stierstorfer for their assistance and the use of their crystal structure and melting point data. 


\section{References Cited}

1 Raevsky, O.A. Physicochemical Descriptors in Property-Based Drug Design Mini Rev. Med. Chem. 2004, 4(10), 1041-1052.

2 Morrill, J. A.; Jensen, R. E.; Madison, P. H.; Chabalowski, C. F. "Prediction of the Formulation Dependence of the Glass Transition Temperatures of Amine-Epoxy Copolymers Using a QSPR Based on the AM1 Method” J. Chem. Inf. Comput. Sci. 2004, 44(3), 912-920.

3 Karthikeyan, M.; Glen, R.C.; Bender, A. General Melting Point Prediction Based on a Diverse Compound Data Set and Artificial Neural Networks J. Chem. Inf. Model 2005, 45(3), 581-590.

4 Bhat,A.U.; Merchant, S.S.; Bhagwat, S.S. Prediction of Melting Points of Organic Compounds Using Extreme Learning Machines Ind. Eng. Chem. Res. 2008, 47(3), 920-925.

5 Nigsch,F.; Bender, A.; van Buuren, B.; Tissen, J.; Nigsch, E; Mitchell, J.B.O. Melting Point Prediction Employing k-Nearest Neighbor Algorithms and Genetic Parameter Optimization J. Chem. Inf. Model. 2006, 46(6), 2412-2422.

6 Paster, I.; Shacham, M.; Brauner, N. Investigation of the Relationships between Molecular Structure, Molecular Descriptors, and Physical Properties Ind. Eng. Chem. Res. 2009, 48(21), 9723-9734.

7 Lazzus, J.A. Hybrid Method to Predict Melting Points of Organic Compounds Using Group Contribution + Neural Network + Particle Swarm Algorithm Ind. Eng. Chem. Res. 2009, 48(18), 8760-8766.

8 Preiss, U.P.; Beichel, W.; Erle, A.M.T.; Paulechka, Y.U.; Krossing, I. Is Universal, Simple Melting Point Prediction Possible? Chem. Phys. Chem. 2011, 12, 2959 - 2972.

9 Salahinejad, M.; Le, T.C.; Winkler, D.A. Capturing the Crystal: Prediction of Enthalpy of Sublimation, Crystal Lattice Energy, and Melting Points of Organic Compounds J. Chem. Inf. Model. 2013, 53, 223-229.

10 Hughes, L.D.; Palmer, D.S.; Nigsch, F.; and Mitchell, J.B.O., Why Are Some Properties More Difficult To Predict than Others? A Study of QSPR Models of Solubility, Melting Point, and Log P. J. Chem. Inf. Model, 2008, 48(1), 220-232.

11. Stewart, James J. P. Optimization of parameters for semiempirical methods. I. Method. J. Comp. Chem. 1989, 10(2), 209-220. 
12. Dewar, M.J.S.; Zoebisch, E.G.; Healy, E.F.; Stewart, J.J.P. The development and use of quantum mechanical molecular models. 76. AMI: a new general purpose quantum mechanical molecular model. J. Am. Chem. Soc. 1985, 107, 3902-3909.

13 Becke A.D. Density-functional exchange-energy approximation with correct asymptotic behavior. Phys. Rev. A 1988, 38, 3098 - 3100.

14. Schäfer, A.; Horn, H.; Ahlrichs, R. Fully optimized contracted Gaussian basis sets for atoms Li to Kr. J. Chem. Phys. 1992, 97, 2571-2577.

15. Vosko, S.; Wilk, L.; Nusair, M. Accurate spin-dependent electron liquid correlation energies for local spin density calculations: a critical analysis. Can. J. Phys. 1980, 58, 1200-1211.

16. Perdew, J.P. Density-functional approximation for the correlation energy of the inhomogeneous electron gas. Phys. Rev. B 1986, 33, $8822-8824$.

17. Schäfer, A.; Huber, C.; Ahlrichs, R. Fully optimized contracted Gaussian basis sets of triple zeta valence quality for atoms Li to Kr. J. Chem. Phys. 1994, 100, 5829-5835

18. Klamt, A.; Schüürmann, G. COSMO: a new approach to dielectric screening in solvents with explicit expressions for the screening energy and its gradient. J. Chem. Soc. Perkin Trans. 1993, 2, 799-805.

${ }^{19}$ Salahinejad, M.; Le, T.C.; Winkler, D.A. Capturing the Crystal: Prediction of Enthalpy of Sublimation, Crystal Lattice Energy, and Melting Points of Organic Compounds. J. Chem. Inf. Model 2013 53, 223-229.

${ }^{20}$ Lazzus, J. Neural Network Based on Quantum Chemistry for the Predicting Melting Point of Organic Compounds. Chin. J. Chem. Phys. 2009, 22(1), 19-26.

21. CODESSA 3, Semichem Inc.: Box 1649 Shawnee, KS 66222.

${ }^{22}$ Yan, C.; Han, M.; Wan, H.; Guan, G. QSAR Correlation of the Melting Points for Imidazolium Bromides and Imidazolium Chlorides Ionic Liquids. Fluid Phase Equil. 2010, 292, 104-109.

${ }^{23}$ Brauner, N.; Shacham, M. Prediction of Normal Melting Point of Pure Substances by a Reference Series Method. AlChE J. 2013, 59(10), 3730-3740. 
${ }^{24}$ Liu, Y.; Holder, A. A quantum mechanical quantitative structure-property relationship study of the melting point of a variety of organosilicons. J. Mol. Graph. Model. 2011, 31, 57-64.

25. Dewar, M.J.S.; Jie, C.; Yu, G. SAM1: the first of a new series of general purpose quantum mechanical molecular models. Tetrahedron 1993, 49, 5003-5038.

26 Keshavarz, M.H.; Pouretedal, H.R. New approach for predicting melting point of carbocyclic nitroaromatic compounds. J. Hazard Mater. 2007, 148, 592-598.

27. Chakka, S.; Boddu, V.M.; Maloney, S.W.; Damavarapu, R. Prediction of Physicohemical Properties of Energetic Materials via EPI Suite. In, Energetic Materials: thermophysical properties, predictions, and experimental measurements. Boddu, V.; Redner, P., eds., CRC Press: Boca Raton, FL: 2010.

${ }^{28}$ Francis, L. Neural Networks Demystified. Casualty Actuary Forum 2001, 253-320.

29 AMPAC 8 with Graphical User Interface, Semichem Inc.: Box 1649 Shawnee, KS 66222.

30 Klapotke, T.M. Ludwig Maxamillian University München. Private Communication, 2013.

31 Morrill, J.A.; Byrd, E.F.C. "Development of Quantitative Structure Property Relationships (QSPRs) for Predictive Modeling and Design of Energetic Materials” J. Mol. Graph. Model. 2008, 27, 349-355.

32 D.A. Belsey, E. Kuh, R.E. Welsche, Regression Diagnostics, Wiley, New York, 1980.

${ }^{33}$ Morrill, J.A.; Byrd, E.F.C. "Development of Quantitative Structure Property Relationships (QSPRs) for Predictive Modeling and Design of Energetic Materials" J. Mol. Graph. Model. 2008, 27, 349-355.

34. StataCorp. Stata Statistical Software: Release 10., StataCorp LP: College Station, TX, 2007.

35. D.T. Stanton, P.C. Jurs, Development and use of charged partial surface area descriptors in computer-assisted quantitative structure activity studies. Anal. Chem., 1990, 62, 2323-2329.

36. D.T. Stanton, L.M. Egolf, P.C. Jurs, M.G. Hicks, Computer-assisted study of the relationship between molecular structurw and surface tension of organic compounds. J. Chem. Inf. Comput. Sci., 1992, 32, 109-115. 
37. Balaban, A.T. Highly discriminating distance-based topological index. Chem. Phys. Lett., 1981, 89, 399-404.

38. A. T. Balaban, Topological indices based on topological distances in molecular graphs. Pure and Appl. Chem., 1983, 55, 199-206.

39. K. Fukui, Theory of Orientation and Stereoselection, Springer-Verlag, Berlin, 1975. 
Graphical Abstract

\section{Graphical Abstract}

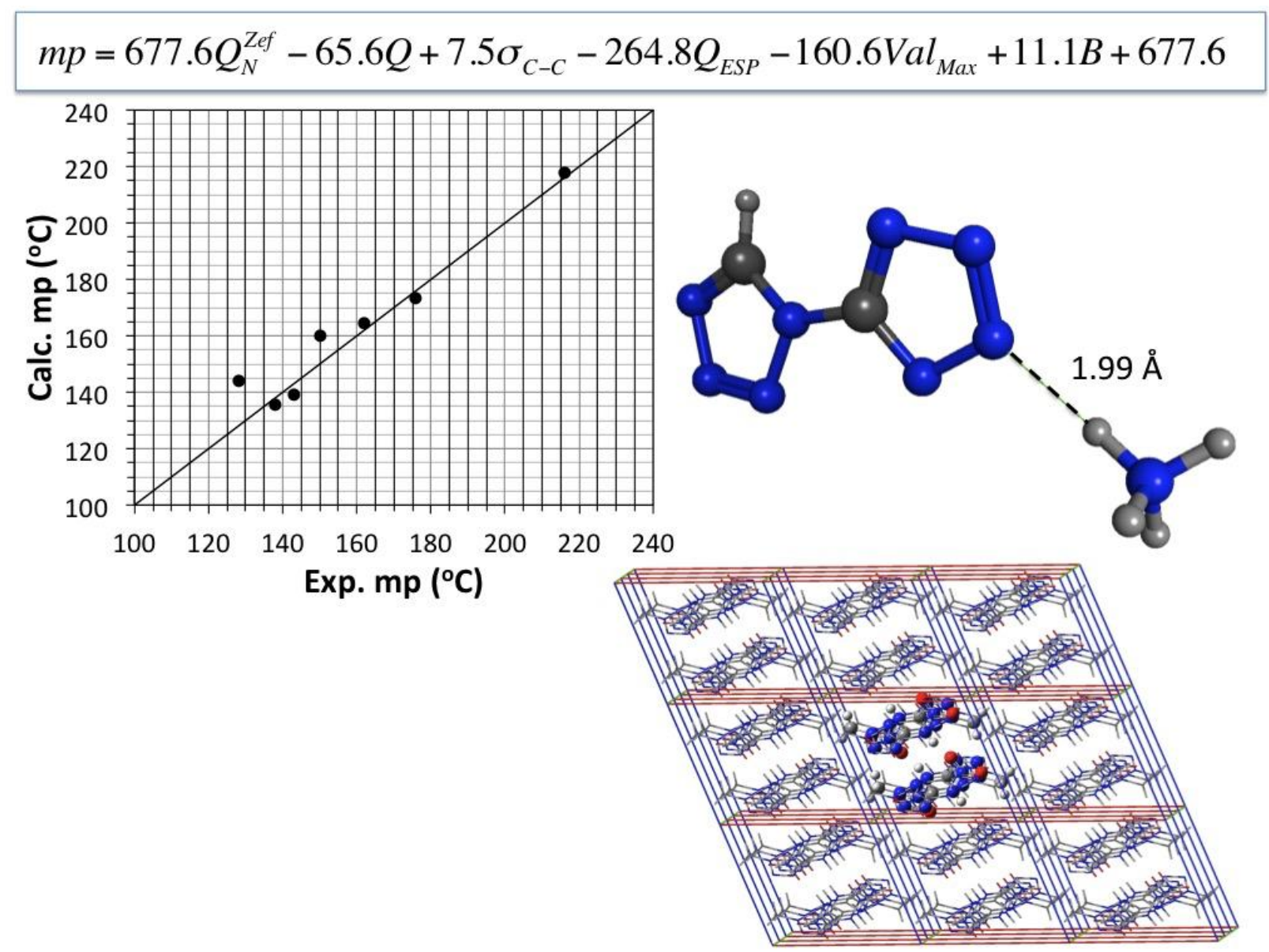

